\title{
Additional information for
}

Cationic Zirconocene and Hafnocene Aryl Complexes

Orson Sydora, Stefan M. Kilyanek and Richard F. Jordan*

Department of Chemistry, The University of Chicago

5735 South Ellis Avenue, Chicago, Illinois, 60637

E-mail: rfjordan@uchicago.edu

\section{Atomic coordinates $\left(x, y, z\right.$, in $\AA$ ) and vibrational frequencies $\left(\mathrm{cm}^{-1}\right)$ for calculated structures.}

\author{
$\mathrm{Cp}_{2} \mathrm{Zr}\left(\mathrm{CH}_{2} \mathrm{C}_{6} \mathrm{H}_{5}\right)\left(\mathrm{ClC}_{6} \mathrm{H}_{5}\right)^{+}(\mathbf{S 1})$
}

Coordinates

$\begin{array}{lrrr} & \mathrm{x} \AA & \mathrm{y} \AA & \mathrm{z} \AA \\ \mathrm{C} & 0.095581000 & -0.155117000 & 0.047388000 \\ \mathrm{C} & 0.065989000 & -0.092834000 & 1.479671000 \\ \mathrm{C} & 1.413557000 & -0.063798000 & 1.942539000 \\ \mathrm{C} & 2.275818000 & -0.066884000 & 0.808152000 \\ \mathrm{C} & 1.456587000 & -0.157862000 & -0.362017000 \\ \mathrm{C} & 0.967788000 & 3.181551000 & -1.618012000 \\ \mathrm{C} & 0.974092000 & 4.286218000 & -0.702935000 \\ \mathrm{C} & 2.234658000 & 4.302597000 & -0.044276000 \\ \mathrm{C} & 3.005614000 & 3.203456000 & -0.522398000 \\ \mathrm{C} & 2.233883000 & 2.532579000 & -1.519869000 \\ \mathrm{C} & 2.044717000 & 2.840793000 & 2.634931000 \\ \mathrm{C} & 0.703563000 & 3.330019000 & 2.990641000 \\ \mathrm{C} & 0.277762000 & 4.662295000 & 2.689093000 \\ \mathrm{C} & -1.008654000 & 5.108389000 & 3.009778000 \\ \mathrm{C} & -1.933415000 & 4.239892000 & 3.617388000 \\ \mathrm{C} & -1.554591000 & 2.921309000 & 3.913620000 \\ \mathrm{C} & -0.266028000 & 2.468194000 & 3.597370000 \\ \mathrm{C} & -2.438056000 & 3.297280000 & -0.999580000 \\ \mathrm{C} & -2.696726000 & 4.671704000 & -0.992980000 \\ \mathrm{C} & -3.241440000 & 5.244006000 & -2.154763000 \\ \mathrm{C} & -3.516161000 & 4.448355000 & -3.277756000 \\ \mathrm{C} & -3.255228000 & 3.069359000 & -3.246597000 \\ \mathrm{C} & -2.711613000 & 2.473186000 & -2.096341000 \\ \mathrm{Cl} & -1.802107000 & 2.553285000 & 0.505875000\end{array}$




$\begin{array}{lrrr}\mathrm{Zr} & 0.996867000 & 2.136606000 & 0.658315000 \\ \mathrm{H} & -0.772118000 & -0.227277000 & -0.608543000 \\ \mathrm{H} & -0.831180000 & -0.117467000 & 2.098740000 \\ \mathrm{H} & 1.735781000 & -0.053077000 & 2.982659000 \\ \mathrm{H} & 3.366387000 & -0.054574000 & 0.831112000 \\ \mathrm{H} & 1.810880000 & -0.254345000 & -1.386909000 \\ \mathrm{H} & 0.156842000 & 2.919331000 & -2.298764000 \\ \mathrm{H} & 0.161898000 & 4.999412000 & -0.561551000 \\ \mathrm{H} & 2.565145000 & 5.030464000 & 0.694938000 \\ \mathrm{H} & 4.021358000 & 2.950565000 & -0.215675000 \\ \mathrm{H} & 2.574547000 & 1.696405000 & -2.127776000 \\ \mathrm{H} & 2.421688000 & 2.036031000 & 3.275276000 \\ \mathrm{H} & 2.801679000 & 3.623544000 & 2.513187000 \\ \mathrm{H} & 1.002527000 & 5.368551000 & 2.273673000 \\ \mathrm{H} & -1.283525000 & 6.150065000 & 2.814586000 \\ \mathrm{H} & -2.933139000 & 4.597318000 & 3.881390000 \\ \mathrm{H} & -2.255842000 & 2.249197000 & 4.417902000 \\ \mathrm{H} & 0.040541000 & 1.465214000 & 3.908349000 \\ \mathrm{H} & -2.496794000 & 5.273783000 & -0.102747000 \\ \mathrm{H} & -3.455806000 & 6.316669000 & -2.172248000 \\ \mathrm{H} & -3.943153000 & 4.902073000 & -4.176545000 \\ \mathrm{H} & -3.481609000 & 2.444448000 & -4.115605000 \\ \mathrm{H} & -2.523416000 & 1.397400000 & -2.055034000\end{array}$

Vibrational Frequencies $\left(\mathrm{cm}^{-1}\right)$

$\begin{array}{lllllll}14.5 & 279.5 & 710.9 & 900.0 & 1074.3 & 1438.1 & 3144.7 \\ 27.2 & 327.4 & 727.6 & 902.6 & 1076.8 & 1441.9 & 3147.2 \\ 39.8 & 329.4 & 743.0 & 934.8 & 1102.2 & 1444.6 & 3149.5 \\ 45.9 & 378.5 & 790.6 & 945.2 & 1126.7 & 1445.3 & 3151.6 \\ 56.7 & 391.7 & 791.6 & 954.0 & 1128.0 & 1448.4 & 3156.5 \\ 66.3 & 402.3 & 796.7 & 971.1 & 1166.7 & 1448.8 & 3181.9 \\ 77.4 & 406.2 & 800.9 & 972.7 & 1166.8 & 1458.6 & 3182.9 \\ 86.6 & 411.0 & 802.3 & 973.5 & 1171.6 & 1469.2 & 3183.7 \\ 96.2 & 462.7 & 805.1 & 983.4 & 1184.0 & 1483.6 & 3184.8 \\ 121.1 & 498.7 & 807.4 & 986.9 & 1226.0 & 1564.8 & 3195.6 \\ 127.3 & 546.9 & 821.9 & 1011.5 & 1254.9 & 1578.1 & 3196.1 \\ 153.5 & 560.8 & 823.2 & 1015.9 & 1256.8 & 1596.0 & 3203.8 \\ 163.5 & 573.9 & 826.2 & 1017.3 & 1297.5 & 1600.0 & 3208.1 \\ 172.6 & 575.4 & 826.8 & 1020.0 & 1317.0 & 3081.4 & 3211.0 \\ 217.6 & 577.7 & 828.2 & 1023.2 & 1352.8 & 3120.1 & 3217.1 \\ 229.4 & 600.2 & 833.3 & 1030.5 & 1357.6 & 3127.2 & \\ 247.6 & 610.9 & 872.3 & 1052.9 & 1375.2 & 3131.4 & \\ 252.0 & 668.9 & 883.2 & 1060.2 & 1377.6 & 3137.6 & \\ 258.2 & 671.4 & 885.2 & 1068.2 & 1383.8 & 3137.9 & \\ 273.1 & 687.2 & 886.8 & 1069.4 & 1388.2 & 3142.0 & \end{array}$


$\mathrm{Cp}^{*}{ }_{2} \mathrm{Zr}(\mathrm{Cl})\left(\mathrm{ClC}_{6} \mathrm{H}_{5}\right)^{+}(\mathbf{S} 2)$

$\begin{array}{crrr}\text { Coordinates } & & & \\ & \mathrm{x} \AA & \mathrm{y} \AA & \mathrm{z} \AA \\ \mathrm{C} & -0.859034000 & 2.396456000 & -1.008727000 \\ \mathrm{C} & 0.496199000 & 2.289730000 & -0.536482000 \\ \mathrm{C} & 0.449131000 & 2.090873000 & 0.886845000 \\ \mathrm{C} & -0.928044000 & 2.057784000 & 1.297163000 \\ \mathrm{C} & -1.741401000 & 2.265760000 & 0.122484000 \\ \mathrm{C} & -1.270383000 & 2.756478000 & -2.411301000 \\ \mathrm{H} & -1.272682000 & 3.856297000 & -2.533230000 \\ \mathrm{H} & -2.285644000 & 2.401837000 & -2.652069000 \\ \mathrm{H} & -0.579988000 & 2.339383000 & -3.160701000 \\ \mathrm{C} & 1.726357000 & 2.535919000 & -1.364919000 \\ \mathrm{H} & 1.615043000 & 2.135094000 & -2.384798000 \\ \mathrm{H} & 2.625759000 & 2.082001000 & -0.920373000 \\ \mathrm{H} & 1.910935000 & 3.623264000 & -1.450542000 \\ \mathrm{C} & 1.615120000 & 2.139539000 & 1.835923000 \\ \mathrm{H} & 1.740798000 & 3.173913000 & 2.208888000 \\ \mathrm{H} & 2.562407000 & 1.850296000 & 1.356368000 \\ \mathrm{H} & 1.463076000 & 1.495151000 & 2.717538000 \\ \mathrm{C} & -1.378317000 & 2.132164000 & 2.733751000 \\ \mathrm{H} & -0.898570000 & 1.371094000 & 3.373417000 \\ \mathrm{H} & -2.466993000 & 2.024187000 & 2.834832000 \\ \mathrm{H} & -1.108644000 & 3.118171000 & 3.156761000 \\ \mathrm{C} & -3.194971000 & 2.656055000 & 0.111177000 \\ \mathrm{H} & -3.272167000 & 3.754853000 & 0.217238000 \\ \mathrm{H} & -3.763171000 & 2.214253000 & 0.943035000 \\ \mathrm{H} & -3.697544000 & 2.389480000 & -0.830999000 \\ \mathrm{C} & -2.600301000 & -1.027197000 & 1.100898000 \\ \mathrm{C} & -2.938923000 & -1.023551000 & -0.303745000 \\ \mathrm{C} & -2.076970000 & -1.970094000 & -0.967416000 \\ \mathrm{C} & -1.169020000 & -2.505030000 & 0.000375000 \\ \mathrm{C} & -1.490112000 & -1.916502000 & 1.285711000 \\ \mathrm{C} & -3.460333000 & -0.499407000 & 2.216703000 \\ \mathrm{H} & -4.018585000 & 0.407075000 & 1.939344000 \\ \mathrm{H} & -2.885816000 & -0.287198000 & 3.131040000 \\ \mathrm{H} & -4.212451000 & -1.267508000 & 2.480808000 \\ \mathrm{C} & -4.181485000 & -0.438828000 & -0.924971000 \\ \mathrm{H} & -4.931848000 & -1.238076000 & -1.072468000 \\ \mathrm{H} & -3.991428000 & 0.005860000 & -1.916529000 \\ \mathrm{H} & -4.645084000 & 0.328293000 & -0.288949000 \\ \mathrm{C} & -2.231097000 & -2.450572000 & -2.382336000 \\ \mathrm{H} & -1.278337000 & -2.803673000 & -2.805106000 \\ \mathrm{H} & -2.625118000 & -1.668599000 & -3.050453000 \\ \mathrm{H} & -2.944379000 & -3.296851000 & -2.405744000 \\ & & & \end{array}$




$\begin{array}{lrrr}\mathrm{C} & -0.187866000 & -3.613555000 & -0.275150000 \\ \mathrm{H} & -0.731848000 & -4.557106000 & -0.465267000 \\ \mathrm{H} & 0.491288000 & -3.792565000 & 0.571863000 \\ \mathrm{H} & 0.426268000 & -3.404902000 & -1.167809000 \\ \mathrm{C} & -0.943536000 & -2.344676000 & 2.625824000 \\ \mathrm{H} & -0.876049000 & -1.508443000 & 3.342100000 \\ \mathrm{H} & 0.053480000 & -2.805587000 & 2.548504000 \\ \mathrm{H} & -1.611636000 & -3.102393000 & 3.076921000 \\ \mathrm{C} & 3.317963000 & -0.667693000 & 0.422540000 \\ \mathrm{C} & 3.611402000 & -0.715977000 & -0.941291000 \\ \mathrm{H} & 2.841617000 & -0.953670000 & -1.679289000 \\ \mathrm{C} & 4.933340000 & -0.434356000 & -1.328117000 \\ \mathrm{H} & 5.192118000 & -0.463419000 & -2.390739000 \\ \mathrm{C} & 5.909783000 & -0.127517000 & -0.367898000 \\ \mathrm{H} & 6.935493000 & 0.085926000 & -0.681924000 \\ \mathrm{C} & 5.575307000 & -0.099053000 & 0.994878000 \\ \mathrm{H} & 6.334794000 & 0.132949000 & 1.747332000 \\ \mathrm{C} & 4.261891000 & -0.373141000 & 1.411550000 \\ \mathrm{H} & 3.991003000 & -0.366792000 & 2.470076000 \\ \mathrm{Cl} & 1.659735000 & -1.122458000 & 0.987465000 \\ \mathrm{Cl} & 0.270747000 & -0.398379000 & -2.418575000 \\ \mathrm{Zr} & -0.609312000 & -0.002500000 & -0.183276000\end{array}$

Vibrational Frequencies $\left(\mathrm{cm}^{-1}\right)$

$\begin{array}{lllllll}19.5 & 182.5 & 382.7 & 729.1 & 1022.8 & 1381.3 & 1463.7 \\ 34.9 & 185.1 & 386.7 & 792.6 & 1024.3 & 1388.3 & 1465.5 \\ 40.5 & 188.9 & 392.5 & 797.0 & 1025.7 & 1394.0 & 1468.3 \\ 48.0 & 192.8 & 394.7 & 803.0 & 1048.6 & 1396.1 & 1470.8 \\ 62.1 & 201.1 & 401.6 & 803.8 & 1062.3 & 1397.0 & 1471.3 \\ 71.2 & 204.7 & 403.3 & 805.7 & 1064.5 & 1398.9 & 1472.6 \\ 92.5 & 208.7 & 457.3 & 890.8 & 1065.8 & 1401.7 & 1477.6 \\ 104.8 & 217.1 & 521.8 & 937.4 & 1069.3 & 1404.7 & 1480.7 \\ 114.1 & 237.6 & 523.0 & 942.6 & 1079.8 & 1406.0 & 1484.4 \\ 116.1 & 248.1 & 530.5 & 946.7 & 1097.9 & 1407.3 & 1484.7 \\ 123.4 & 270.8 & 530.8 & 948.9 & 1100.1 & 1409.1 & 1489.4 \\ 125.4 & 277.0 & 540.3 & 952.0 & 1152.0 & 1417.9 & 1490.0 \\ 134.7 & 277.3 & 542.4 & 965.9 & 1153.1 & 1421.5 & 1493.1 \\ 138.4 & 284.6 & 581.2 & 970.9 & 1158.9 & 1428.2 & 1494.3 \\ 144.4 & 285.9 & 584.1 & 1010.8 & 1160.0 & 1435.7 & 1496.0 \\ 148.2 & 288.5 & 587.7 & 1014.0 & 1166.6 & 1439.0 & 1497.9 \\ 156.3 & 289.3 & 590.0 & 1015.0 & 1175.5 & 1442.1 & 1500.8 \\ 159.1 & 290.6 & 594.5 & 1016.4 & 1302.7 & 1449.0 & 1574.2 \\ 161.6 & 294.4 & 598.2 & 1017.0 & 1352.6 & 1450.3 & 1601.2 \\ 162.7 & 326.3 & 598.5 & 1018.9 & 1376.0 & 1457.3 & 2974.8 \\ 173.1 & 339.3 & 660.0 & 1019.1 & 1378.0 & 1458.9 & 2975.7 \\ 178.6 & 366.5 & 666.6 & 1021.2 & 1379.9 & 1462.0 & 2976.6\end{array}$


2977.0

2977.5

2977.7

2980.1

2980.3

2980.7

2981.9

3030.8

3036.4

3038.1

3040.1

3041.0

3044.4

3049.8

3050.6

3050.8

3052.5

3073.5

3081.0

3081.4

3081.6

3084.1

3086.1

3089.6

3092.7

3098.5

3100.2

3131.6

3139.1

3150.0

3157.3

3158.8 
$\mathrm{Cp}_{2} \mathrm{Zr}(\mathrm{Et})\left(\mathrm{PMe}_{3}\right)^{+}$, exo isomer (S3-exo)

$\begin{array}{lrrr}\text { Coordinates } & & & \\ & \mathrm{x} \AA & \mathrm{y} \AA & \mathrm{z} \AA \\ \mathrm{Zr} & -0.088419000 & -0.248141000 & 0.336085000 \\ \mathrm{C} & -0.006628000 & -0.078729000 & 2.892245000 \\ \mathrm{C} & 1.323193000 & -0.377471000 & 2.461753000 \\ \mathrm{C} & 1.806084000 & 0.733771000 & 1.711502000 \\ \mathrm{C} & 0.777068000 & 1.738063000 & 1.695647000 \\ \mathrm{C} & -0.323800000 & 1.241503000 & 2.442250000 \\ \mathrm{H} & -0.636590000 & -0.705912000 & 3.525381000 \\ \mathrm{H} & 1.880520000 & -1.285678000 & 2.693308000 \\ \mathrm{H} & 2.811927000 & 0.832984000 & 1.303317000 \\ \mathrm{H} & 0.841114000 & 2.723864000 & 1.236300000 \\ \mathrm{H} & -1.255741000 & 1.773577000 & 2.639065000 \\ \mathrm{C} & 0.117539000 & -2.101490000 & -1.457109000 \\ \mathrm{C} & 0.785244000 & -2.578279000 & -0.284445000 \\ \mathrm{C} & -0.199203000 & -2.762936000 & 0.732030000 \\ \mathrm{C} & -1.463849000 & -2.367382000 & 0.203186000 \\ \mathrm{C} & -1.265285000 & -1.954469000 & -1.157160000 \\ \mathrm{H} & 0.576708000 & -1.934199000 & -2.431470000 \\ \mathrm{H} & 1.846452000 & -2.817865000 & -0.202486000 \\ \mathrm{H} & -0.019203000 & -3.152272000 & 1.734328000 \\ \mathrm{H} & -2.421796000 & -2.428299000 & 0.721925000 \\ \mathrm{H} & -2.042192000 & -1.635369000 & -1.852385000 \\ \mathrm{C} & -1.326908000 & 1.276099000 & -0.869171000 \\ \mathrm{H} & -1.025227000 & 2.328123000 & -0.779743000 \\ \mathrm{H} & -1.518832000 & 1.013698000 & -1.916167000 \\ \mathrm{C} & -2.448334000 & 0.882747000 & 0.061785000 \\ \mathrm{H} & -2.819978000 & 1.703432000 & 0.693748000 \\ \mathrm{H} & -3.288627000 & 0.381145000 & -0.442568000 \\ \mathrm{H} & -2.155742000 & 0.090328000 & 0.860053000 \\ \mathrm{P} & 1.625906000 & 0.727827000 & -1.600565000 \\ \mathrm{C} & 0.941680000 & 0.914390000 & -3.315513000 \\ \mathrm{H} & 1.747809000 & 1.212909000 & -4.007065000 \\ \mathrm{H} & 0.494423000 & -0.024094000 & -3.677997000 \\ \mathrm{H} & 0.164769000 & 1.694613000 & -3.322916000 \\ \mathrm{C} & 2.297027000 & 2.434371000 & -1.320397000 \\ \mathrm{H} & 1.470563000 & 3.156515000 & -1.224219000 \\ \mathrm{H} & 2.910431000 & 2.474150000 & -0.406768000 \\ \mathrm{H} & 2.926481000 & 2.730909000 & -2.176606000 \\ \mathrm{C} & 3.179981000 & -0.242153000 & -1.911833000 \\ \mathrm{H} & 3.803419000 & 0.266402000 & -2.666921000 \\ \mathrm{H} & 3.764266000 & -0.349340000 & -0.983567000 \\ \mathrm{H} & 2.935391000 & -1.250332000 & -2.283806000 \\ & & & \\ & & & \end{array}$




\begin{tabular}{|c|c|c|}
\hline \multicolumn{3}{|c|}{ Vibrational Frequencies $\left(\mathrm{cm}^{-1}\right)$} \\
\hline 31.2 & 827.7 & 1458.0 \\
\hline 55.3 & 840.7 & 1465.7 \\
\hline 77.9 & 845.2 & 1535.2 \\
\hline 99.3 & 867.8 & 2433.5 \\
\hline 108.0 & 870.2 & 2990.2 \\
\hline 125.2 & 882.0 & 2994.0 \\
\hline 137.6 & 883.9 & 2997.3 \\
\hline 153.9 & 889.1 & 3025.7 \\
\hline 159.4 & 895.4 & 3057.2 \\
\hline 167.5 & 945.5 & 3075.3 \\
\hline 178.7 & 950.2 & 3077.9 \\
\hline 181.5 & 956.4 & 3081.7 \\
\hline 196.8 & 1009.7 & 3084.5 \\
\hline 211.1 & 1010.9 & 3091.2 \\
\hline 214.8 & 1020.3 & 3092.0 \\
\hline 246.6 & 1023.4 & 3096.2 \\
\hline 250.0 & 1038.4 & 3126.9 \\
\hline 253.4 & 1058.9 & 3174.6 \\
\hline 262.6 & 1063.7 & 3176.4 \\
\hline 265.4 & 1065.8 & 3178.3 \\
\hline 269.4 & 1067.7 & 3180.9 \\
\hline 293.7 & 1069.2 & 3190.2 \\
\hline 315.9 & 1122.2 & 3190.3 \\
\hline 336.6 & 1123.3 & 3195.1 \\
\hline 354.2 & 1166.5 & 3196.2 \\
\hline 383.9 & 1255.6 & 3203.6 \\
\hline 429.9 & 1257.3 & 3207.3 \\
\hline 569.1 & 1304.0 & \\
\hline 573.4 & 1308.1 & \\
\hline 576.3 & 1330.7 & \\
\hline 581.2 & 1372.9 & \\
\hline 609.1 & 1374.2 & \\
\hline 640.1 & 1377.2 & \\
\hline 700.2 & 1380.3 & \\
\hline 704.8 & 1394.5 & \\
\hline 784.4 & 1420.3 & \\
\hline 787.4 & 1432.2 & \\
\hline 790.2 & 1436.2 & \\
\hline 796.5 & 1437.7 & \\
\hline 803.1 & 1442.8 & \\
\hline 803.3 & 1443.4 & \\
\hline 814.4 & 1446.6 & \\
\hline 823.9 & 1446.8 & \\
\hline 824.1 & 1448.4 & \\
\hline 825.9 & 1453.3 & \\
\hline
\end{tabular}


$\mathrm{Cp}_{2} \mathrm{Zr}(\mathrm{Et})\left(\mathrm{PMe}_{3}\right)^{+}$, endo isomer (S3-endo)

$\begin{array}{lccc}\text { Coordinates } & & & \\ & \mathrm{x} \AA & \mathrm{y} \AA & \mathrm{z} \AA \\ \mathrm{Zr} & -0.049208000 & 0.073280000 & -0.126426000 \\ \mathrm{C} & 0.031160000 & -0.006054000 & 2.392374000 \\ \mathrm{C} & 1.396065000 & 0.028144000 & 1.957190000 \\ \mathrm{C} & 1.633288000 & 1.294564000 & 1.357995000 \\ \mathrm{C} & 0.415227000 & 2.050426000 & 1.420580000 \\ \mathrm{C} & -0.562069000 & 1.253298000 & 2.084154000 \\ \mathrm{H} & -0.456205000 & -0.829356000 & 2.916152000 \\ \mathrm{H} & 2.128663000 & -0.769942000 & 2.080443000 \\ \mathrm{H} & 2.590800000 & 1.643994000 & 0.971091000 \\ \mathrm{H} & 0.278517000 & 3.077851000 & 1.080748000 \\ \mathrm{H} & -1.574933000 & 1.560356000 & 2.337602000 \\ \mathrm{C} & 0.678330000 & -1.660761000 & -1.894538000 \\ \mathrm{C} & 1.245677000 & -2.035161000 & -0.638863000 \\ \mathrm{C} & 0.168318000 & -2.412007000 & 0.226379000 \\ \mathrm{C} & -1.054898000 & -2.239617000 & -0.483007000 \\ \mathrm{C} & -0.739273000 & -1.760078000 & -1.791998000 \\ \mathrm{H} & 1.235422000 & -1.398309000 & -2.793748000 \\ \mathrm{H} & 2.308852000 & -2.092964000 & -0.403570000 \\ \mathrm{H} & 0.269351000 & -2.797343000 & 1.241122000 \\ \mathrm{H} & -2.051253000 & -2.455149000 & -0.098929000 \\ \mathrm{H} & -1.456946000 & -1.542294000 & -2.584646000 \\ \mathrm{P} & 1.598082000 & 1.445799000 & -1.932007000 \\ \mathrm{C} & 1.010415000 & 1.469289000 & -3.692060000 \\ \mathrm{H} & 1.723356000 & 2.022570000 & -4.326349000 \\ \mathrm{H} & 0.903643000 & 0.447113000 & -4.087170000 \\ \mathrm{H} & 0.028029000 & 1.965970000 & -3.751472000 \\ \mathrm{C} & 1.841958000 & 3.256993000 & -1.612155000 \\ \mathrm{H} & 0.872311000 & 3.780510000 & -1.638536000 \\ \mathrm{H} & 2.301456000 & 3.423416000 & -0.625289000 \\ \mathrm{H} & 2.498013000 & 3.693799000 & -2.384131000 \\ \mathrm{C} & 3.336963000 & 0.822325000 & -2.075256000 \\ \mathrm{H} & 3.909365000 & 1.448727000 & -2.779996000 \\ \mathrm{H} & 3.834534000 & 0.846493000 & -1.092467000 \\ \mathrm{H} & 3.343435000 & -0.217061000 & -2.439299000 \\ \mathrm{C} & -2.328853000 & 0.479200000 & -0.197906000 \\ \mathrm{H} & -2.856299000 & 0.735950000 & 0.726687000 \\ \mathrm{H} & -2.887377000 & -0.298561000 & -0.732384000 \\ \mathrm{C} & -2.003104000 & 1.671755000 & -1.070176000 \\ \mathrm{H} & -2.223446000 & 2.642674000 & -0.599407000 \\ \mathrm{H} & -0.879402000 & 1.770820000 & -1.324275000 \\ \mathrm{H} & -2.459012000 & 1.634801000 & -2.072991000 \\ & & & \\ & & & \end{array}$




\begin{tabular}{|c|c|c|}
\hline \multicolumn{3}{|c|}{ Vibrational Frequencies $\left(\mathrm{cm}^{-}\right)$} \\
\hline 32.0 & 827.8 & 1459.8 \\
\hline 55.3 & 840.2 & 1465.8 \\
\hline 75.9 & 842.5 & 1515.2 \\
\hline 87.7 & 858.2 & 2481.5 \\
\hline 91.5 & 875.5 & 2992.3 \\
\hline 102.5 & 881.3 & 2992.8 \\
\hline 129.0 & 882.4 & 2995.3 \\
\hline 130.7 & 893.3 & 3015.7 \\
\hline 143.6 & 921.0 & 3074.6 \\
\hline 147.6 & 948.9 & 3076.0 \\
\hline 167.2 & 949.9 & 3077.1 \\
\hline 176.7 & 957.8 & 3081.1 \\
\hline 186.7 & 1008.0 & 3081.4 \\
\hline 195.2 & 1015.5 & 3089.7 \\
\hline 234.6 & 1021.5 & 3090.3 \\
\hline 243.8 & 1022.4 & 3091.9 \\
\hline 247.0 & 1027.4 & 3138.2 \\
\hline 249.5 & 1061.3 & 3175.7 \\
\hline 253.8 & 1065.4 & 3177.1 \\
\hline 272.0 & 1068.4 & 3180.6 \\
\hline 277.9 & 1071.3 & 3182.7 \\
\hline 287.4 & 1079.5 & 3190.5 \\
\hline 295.7 & 1124.5 & 3192.5 \\
\hline 307.3 & 1125.2 & 3197.4 \\
\hline 335.2 & 1162.3 & 3199.3 \\
\hline 342.4 & 1255.2 & 3208.1 \\
\hline 440.0 & 1258.2 & 3214 . \\
\hline 571.6 & 1307.5 & \\
\hline 575.4 & 1308.3 & \\
\hline 578.4 & 1331.0 & \\
\hline 582.3 & 1374.9 & \\
\hline 642.1 & 1377.7 & \\
\hline 681.0 & 1380.7 & \\
\hline 703.1 & 1384.3 & \\
\hline 711.5 & 1408.3 & \\
\hline 789.5 & 1417.8 & \\
\hline 790.6 & 1431.1 & \\
\hline 793.3 & 1435.8 & \\
\hline 800.9 & 1437.7 & \\
\hline 805.4 & 1439.7 & \\
\hline 806.9 & 1442.0 & \\
\hline 820.1 & 1444.8 & \\
\hline 821.7 & 1447.1 & \\
\hline 823.4 & 1448.5 & \\
\hline 826.7 & 1451.0 & \\
\hline
\end{tabular}




$\begin{array}{lrcc}\mathrm{Cp}_{2} \mathrm{Zr}(\mathrm{Et})(\mathrm{THF})^{+}(\mathbf{S} 4) & & \\ & & & \\ \text { Coordinates } & & & \\ & \mathrm{x} \AA & \mathrm{y} \AA & \mathrm{z} \AA \\ \mathrm{Zr} & 0.245534000 & -0.222313000 & 0.077803000 \\ \mathrm{C} & 0.323110000 & -0.108745000 & 2.587743000 \\ \mathrm{C} & 1.669275000 & -0.347892000 & 2.153376000 \\ \mathrm{C} & 2.124972000 & 0.826476000 & 1.487160000 \\ \mathrm{C} & 1.062210000 & 1.776934000 & 1.484409000 \\ \mathrm{C} & -0.048971000 & 1.204235000 & 2.175281000 \\ \mathrm{H} & -0.292527000 & -0.794912000 & 3.170260000 \\ \mathrm{H} & 2.257740000 & -1.246505000 & 2.342061000 \\ \mathrm{H} & 3.115432000 & 0.965373000 & 1.049759000 \\ \mathrm{H} & 1.096278000 & 2.781489000 & 1.059759000 \\ \mathrm{H} & -1.002202000 & 1.693537000 & 2.372639000 \\ \mathrm{C} & -0.297608000 & -2.117454000 & -1.541788000 \\ \mathrm{C} & 0.621506000 & -2.643696000 & -0.577426000 \\ \mathrm{C} & -0.038519000 & -2.669581000 & 0.684639000 \\ \mathrm{C} & -1.358159000 & -2.147572000 & 0.505801000 \\ \mathrm{C} & -1.522307000 & -1.826909000 & -0.873079000 \\ \mathrm{H} & -0.116045000 & -1.999589000 & -2.611093000 \\ \mathrm{H} & 1.634711000 & -2.999530000 & -0.772747000 \\ \mathrm{H} & 0.383458000 & -3.039782000 & 1.619060000 \\ \mathrm{H} & -2.117911000 & -2.040673000 & 1.281546000 \\ \mathrm{H} & -2.427439000 & -1.436206000 & -1.336067000 \\ \mathrm{C} & -1.497685000 & 1.116860000 & -0.464163000 \\ \mathrm{H} & -1.045769000 & 2.126178000 & -0.355461000 \\ \mathrm{H} & -1.516819000 & 0.888118000 & -1.556873000 \\ \mathrm{C} & -2.933810000 & 1.154301000 & 0.070063000 \\ \mathrm{H} & -2.960269000 & 1.414619000 & 1.142504000 \\ \mathrm{H} & -3.551440000 & 1.903856000 & -0.458687000 \\ \mathrm{H} & -3.441039000 & 0.180060000 & -0.039748000 \\ \mathrm{O} & 1.625411000 & 0.442278000 & -1.605899000 \\ \mathrm{C} & 1.782621000 & 1.874777000 & -1.971409000 \\ \mathrm{H} & 2.380067000 & 2.357074000 & -1.178919000 \\ \mathrm{H} & 0.773761000 & 2.312518000 & -2.002990000 \\ \mathrm{C} & 2.563497000 & -0.376576000 & -2.421699000 \\ \mathrm{H} & 3.106784000 & -1.039865000 & -1.732692000 \\ \mathrm{H} & 1.949485000 & -0.975376000 & -3.113225000 \\ \mathrm{C} & 2.510249000 & 1.848417000 & -3.31145000 \\ \mathrm{H} & 1.797844000 & 1.691152000 & -4.138716000 \\ \mathrm{H} & 3.053925000 & 2.787870000 & -3.496619000 \\ \mathrm{C} & 3.446792000 & 0.635780000 & -3.149224000 \\ \mathrm{H} & 4.326258000 & 0.900021000 & -2.537652000 \\ \mathrm{H} & 3.805650000 & 0.240028000 & -4.112104000\end{array}$




\begin{tabular}{|c|c|c|}
\hline \multicolumn{3}{|c|}{ Vibrational Frequencies $\left(\mathrm{cm}^{-}\right)$} \\
\hline 38.9 & 877.9 & 1475.8 \\
\hline 45.6 & 885.0 & 1480.4 \\
\hline 52.7 & 895.2 & 1490.1 \\
\hline 62.8 & 907.0 & 1502.7 \\
\hline 73.0 & 912.0 & 2870.6 \\
\hline 100.7 & 917.9 & 2942.9 \\
\hline 115.4 & 952.0 & 2968.4 \\
\hline 126.9 & 981.1 & 3004.5 \\
\hline 130.5 & 1009.5 & 3012.1 \\
\hline 150.5 & 1013.7 & 3018.0 \\
\hline 166.2 & 1014.7 & 3020.2 \\
\hline 184.2 & 1021.0 & 3027.9 \\
\hline 194.9 & 1023.1 & 3045.8 \\
\hline 220.8 & 1036.1 & 3070.4 \\
\hline 241.3 & 1065.3 & 3073.7 \\
\hline 244.5 & 1065.4 & 3082.6 \\
\hline 251.6 & 1068.4 & 3088.0 \\
\hline 265.2 & 1069.8 & 3168.1 \\
\hline 268.2 & 1124.3 & 3171.6 \\
\hline 281.2 & 1126.6 & 3178.0 \\
\hline 320.9 & 1132.3 & 3179.6 \\
\hline 345.7 & 1148.6 & 3185.5 \\
\hline 390.9 & 1156.1 & 3186.5 \\
\hline 560.3 & 1164.1 & 3193.1 \\
\hline 568.8 & 1225.3 & 3198.7 \\
\hline 569.5 & 1229.4 & 3205.7 \\
\hline 573.9 & 1244.9 & 3208.2 \\
\hline 577.5 & 1256.5 & \\
\hline 644.2 & 1258.4 & \\
\hline 672.2 & 1294.2 & \\
\hline 788.4 & 1330.6 & \\
\hline 792.9 & 1338.1 & \\
\hline 796.1 & 1356.1 & \\
\hline 802.2 & 1375.3 & \\
\hline 804.7 & 1377.1 & \\
\hline 814.4 & 1378.7 & \\
\hline 820.9 & 1384.4 & \\
\hline 823.4 & 1393.6 & \\
\hline 825.1 & 1421.5 & \\
\hline 827.3 & 1439.5 & \\
\hline 828.0 & 1441.0 & \\
\hline 831.3 & 1444.6 & \\
\hline 847.4 & 1446.4 & \\
\hline 867.0 & 1467.6 & \\
\hline 871.2 & 1475.4 & \\
\hline
\end{tabular}


$\mathrm{Cp}_{2} \mathrm{Zr}(\mathrm{Ph})\left(\mathrm{ClCH}_{2} \mathrm{Cl}\right)^{+}$, endo isomer $\left(\mathbf{2} \mathbf{d} \cdot \mathbf{C H}_{2} \mathbf{C l}_{\mathbf{2}}\right.$-endo $)$

$\begin{array}{lrrr}\text { Coordinates } & & \\ & \mathrm{x} \AA & \mathrm{y} \AA & \mathrm{z} \AA \\ \mathrm{Zr} & 0.013051000 & 0.000359000 & -0.006815000 \\ \mathrm{C} & -0.006331000 & 0.000663000 & 2.491070000 \\ \mathrm{C} & 1.381919000 & -0.008390000 & 2.126988000 \\ \mathrm{C} & 1.678450000 & 1.245301000 & 1.523033000 \\ \mathrm{C} & 0.478653000 & 2.025208000 & 1.495314000 \\ \mathrm{C} & -0.556507000 & 1.267308000 & 2.118395000 \\ \mathrm{H} & -0.532303000 & -0.793443000 & 3.022268000 \\ \mathrm{H} & 2.095509000 & -0.809131000 & 2.321011000 \\ \mathrm{H} & 2.661492000 & 1.567718000 & 1.174180000 \\ \mathrm{H} & 0.379155000 & 3.036206000 & 1.097086000 \\ \mathrm{H} & -1.578714000 & 1.599956000 & 2.289371000 \\ \mathrm{C} & -0.185010000 & -1.780417000 & -1.787755000 \\ \mathrm{C} & 0.937993000 & -2.155229000 & -0.973638000 \\ \mathrm{C} & 0.452848000 & -2.479947000 & 0.320787000 \\ \mathrm{C} & -0.969591000 & -2.298359000 & 0.318456000 \\ \mathrm{C} & -1.363114000 & -1.902316000 & -0.994913000 \\ \mathrm{H} & -0.162942000 & -1.501229000 & -2.842732000 \\ \mathrm{H} & 1.982764000 & -2.197882000 & -1.286680000 \\ \mathrm{H} & 1.054037000 & -2.824139000 & 1.162163000 \\ \mathrm{H} & -1.641242000 & -2.493889000 & 1.155343000 \\ \mathrm{H} & -2.381321000 & -1.712069000 & -1.329071000 \\ \mathrm{Cl} & 2.198305000 & 0.984060000 & -1.534011000 \\ \mathrm{C} & -1.989325000 & 0.851302000 & -0.369695000 \\ \mathrm{C} & -3.367144000 & 0.803906000 & -0.094183000 \\ \mathrm{C} & -4.252928000 & 1.635896000 & -0.797734000 \\ \mathrm{C} & -3.774262000 & 2.519182000 & -1.783636000 \\ \mathrm{C} & -2.403919000 & 2.582469000 & -2.067894000 \\ \mathrm{C} & -1.524335000 & 1.750538000 & -1.345033000 \\ \mathrm{H} & -5.325299000 & 1.594959000 & -0.580143000 \\ \mathrm{H} & -4.472329000 & 3.162907000 & -2.327198000 \\ \mathrm{H} & -2.020102000 & 3.273207000 & -2.824957000 \\ \mathrm{H} & -0.440951000 & 1.854701000 & -1.584378000 \\ \mathrm{C} & 2.294187000 & 0.522955000 & -3.332266000 \\ \mathrm{H} & 3.212593000 & 1.014266000 & -3.676930000 \\ \mathrm{H} & 2.367165000 & -0.570598000 & -3.361712000 \\ \mathrm{Cl} & 0.913661000 & 1.073680000 & -4.276175000 \\ \mathrm{H} & -3.759745000 & 0.117315000 & 0.664835000\end{array}$

Vibrational Frequencies $\left(\mathrm{cm}^{-1}\right)$

17.0

25.3

33.4
40.9

52.7

61.5
70.5

79.1 107.8
112.7

138.1

145.8
179.0

189.8

242.3
247.5

257.2

274.2
285.1

313.9

325.0 


$\begin{array}{llrrrrr}341.7 & 743.0 & 867.8 & 1019.6 & 1184.5 & 1433.6 & 3146.0 \\ 367.3 & 784.9 & 874.8 & 1027.2 & 1256.4 & 1436.7 & 3157.3 \\ 449.1 & 795.7 & 880.9 & 1032.6 & 1260.7 & 1442.4 & 3170.6 \\ 563.6 & 798.7 & 890.0 & 1062.0 & 1269.4 & 1448.1 & 3171.8 \\ 570.2 & 808.1 & 894.3 & 1066.6 & 1295.7 & 1478.5 & 3181.3 \\ 575.6 & 810.6 & 898.5 & 1069.6 & 1346.7 & 1565.9 & 3184.1 \\ 576.0 & 815.5 & 928.0 & 1072.6 & 1368.4 & 1578.1 & 3187.1 \\ 579.7 & 820.9 & 967.3 & 1085.5 & 1372.0 & 2909.8 & 3189.6 \\ 599.2 & 822.4 & 968.0 & 1121.2 & 1376.1 & 3070.4 & 3199.0 \\ 638.7 & 825.1 & 1005.9 & 1123.0 & 1382.0 & 3102.2 & 3202.9 \\ 668.7 & 827.0 & 1009.4 & 1154.2 & 1418.5 & 3124.3 & 3217.5 \\ 699.2 & 830.1 & 1011.4 & 1165.5 & 1426.0 & 3131.5 & 3218.6\end{array}$


$\mathrm{Cp}_{2} \mathrm{Zr}(\mathrm{Ph})\left(\mathrm{ClCH}_{2} \mathrm{Cl}\right)^{+}$, exo isomer $\left(\mathbf{2} \mathbf{d} \cdot \mathbf{C H}_{2} \mathbf{C l}_{2}-\mathbf{e x o}\right)$

$\begin{array}{lrrr}\text { Coordinates } & & \\ & \mathrm{x} \AA & \mathrm{y} \AA & \mathrm{z} \AA \\ \mathrm{Zr} & 0.000303000 & -0.000348000 & -0.000395000 \\ \mathrm{C} & -0.000251000 & -0.000547000 & 2.231096000 \\ \mathrm{C} & 0.613002000 & -0.001490000 & 3.497004000 \\ \mathrm{C} & -0.177237000 & -0.028218000 & 4.658831000 \\ \mathrm{C} & -1.580484000 & -0.057016000 & 4.571775000 \\ \mathrm{C} & -2.213694000 & -0.061080000 & 3.319807000 \\ \mathrm{C} & -1.398318000 & -0.032803000 & 2.173890000 \\ \mathrm{H} & 1.704020000 & 0.018421000 & 3.593644000 \\ \mathrm{H} & 0.304607000 & -0.028267000 & 5.641921000 \\ \mathrm{H} & -2.185751000 & -0.079090000 & 5.483093000 \\ \mathrm{H} & -3.304480000 & -0.087336000 & 3.239071000 \\ \mathrm{H} & -1.950951000 & -0.047640000 & 1.191638000 \\ \mathrm{Cl} & 2.665083000 & 0.141962000 & 0.874001000 \\ \mathrm{C} & 4.154060000 & -0.103978000 & -0.207707000 \\ \mathrm{Cl} & 3.836395000 & 0.022237000 & -1.941589000 \\ \mathrm{H} & 4.854050000 & 0.682262000 & 0.100291000 \\ \mathrm{H} & 4.526035000 & -1.108234000 & 0.028104000 \\ \mathrm{C} & -1.104465000 & 2.321500000 & -0.001151000 \\ \mathrm{C} & 0.296607000 & 2.537289000 & 0.029992000 \\ \mathrm{C} & 0.839322000 & 2.070220000 & -1.217744000 \\ \mathrm{C} & -0.230025000 & 1.561888000 & -2.004290000 \\ \mathrm{C} & -1.434511000 & 1.672667000 & -1.236461000 \\ \mathrm{H} & -1.803044000 & 2.566620000 & 0.799790000 \\ \mathrm{H} & 0.855523000 & 2.998128000 & 0.844567000 \\ \mathrm{H} & 1.883817000 & 2.128102000 & -1.524431000 \\ \mathrm{H} & -0.147407000 & 1.152610000 & -3.011398000 \\ \mathrm{H} & -2.438157000 & 1.407929000 & -1.573335000 \\ \mathrm{C} & -1.014051000 & -1.631580000 & -1.661920000 \\ \mathrm{C} & 0.399839000 & -1.686145000 & -1.869209000 \\ \mathrm{C} & 0.987485000 & -2.263410000 & -0.701575000 \\ \mathrm{C} & -0.045958000 & -2.536999000 & 0.240690000 \\ \mathrm{C} & -1.287967000 & -2.156652000 & -0.362453000 \\ \mathrm{H} & -1.755954000 & -1.283135000 & -2.381216000 \\ \mathrm{H} & 0.931702000 & -1.390014000 & -2.773484000 \\ \mathrm{H} & 2.047965000 & -2.471527000 & -0.559998000 \\ \mathrm{H} & 0.080439000 & -2.982160000 & 1.226879000 \\ \mathrm{H} & -2.276427000 & -2.275907000 & 0.084515000\end{array}$

Vibrational Frequencies $\left(\mathrm{cm}^{-1}\right)$

$\begin{array}{ccccccc}9.2 & 42.0 & 77.9 & 119.5 & 170.4 & 245.5 & 289.5 \\ 29.5 & 56.1 & 84.6 & 123.1 & 185.6 & 254.8 & 302.4 \\ 30.5 & 59.1 & 108.8 & 140.6 & 243.5 & 268.0 & 320.2\end{array}$




$\begin{array}{llrllll}338.3 & 737.6 & 869.8 & 1018.1 & 1197.2 & 1434.2 & 3146.9 \\ 373.2 & 777.0 & 880.6 & 1021.8 & 1255.0 & 1438.0 & 3156.3 \\ 433.0 & 794.8 & 883.4 & 1031.3 & 1256.6 & 1440.3 & 3176.8 \\ 567.1 & 800.3 & 885.9 & 1060.6 & 1270.7 & 1449.1 & 3178.0 \\ 571.3 & 803.6 & 889.5 & 1063.5 & 1300.4 & 1486.0 & 3185.3 \\ 573.8 & 807.9 & 895.3 & 1066.2 & 1351.0 & 1560.0 & 3186.3 \\ 577.4 & 814.9 & 925.9 & 1067.8 & 1368.5 & 1586.4 & 3193.8 \\ 580.7 & 821.1 & 962.6 & 1095.5 & 1371.4 & 2764.7 & 3194.6 \\ 600.1 & 822.4 & 968.5 & 1121.7 & 1374.6 & 3069.7 & 3198.2 \\ 630.4 & 825.0 & 999.7 & 1122.8 & 1376.8 & 3110.6 & 3202.5 \\ 661.7 & 825.7 & 1007.5 & 1152.8 & 1416.7 & 3126.5 & 3207.5 \\ 692.9 & 829.2 & 1012.3 & 1166.5 & 1433.5 & 3134.1 & 3212.5\end{array}$


$\mathrm{Cp}_{2} \mathrm{Zr}(o$-tolyl $)\left(\mathrm{ClCH}_{2} \mathrm{Cl}\right)^{+}$, endo isomer $\left(\mathbf{2} \mathbf{a} \cdot \mathbf{C H}_{2} \mathbf{C l}_{2}-\mathbf{e n d o}\right)$

$\begin{array}{lrrr}\text { Coordinates } & & \\ & \mathrm{x} \AA & \mathrm{y} \AA & \mathrm{z} \AA \\ \mathrm{Zr} & 0.155272000 & 0.161049000 & 0.038199000 \\ \mathrm{C} & 0.005014000 & 0.038908000 & 2.528869000 \\ \mathrm{C} & 1.409228000 & -0.001644000 & 2.235975000 \\ \mathrm{C} & 1.778583000 & 1.269132000 & 1.712999000 \\ \mathrm{C} & 0.608791000 & 2.092192000 & 1.664343000 \\ \mathrm{C} & -0.482103000 & 1.341574000 & 2.193570000 \\ \mathrm{H} & -0.573723000 & -0.762005000 & 2.990295000 \\ \mathrm{H} & 2.084464000 & -0.836626000 & 2.422424000 \\ \mathrm{H} & 2.788969000 & 1.573897000 & 1.433169000 \\ \mathrm{H} & 0.563378000 & 3.124282000 & 1.313450000 \\ \mathrm{H} & -1.498850000 & 1.704597000 & 2.332276000 \\ \mathrm{C} & -0.000078000 & -1.519408000 & -1.842521000 \\ \mathrm{C} & 1.066581000 & -1.977911000 & -0.996083000 \\ \mathrm{C} & 0.506240000 & -2.351646000 & 0.253632000 \\ \mathrm{C} & -0.907252000 & -2.121793000 & 0.190838000 \\ \mathrm{C} & -1.220437000 & -1.643376000 & -1.116766000 \\ \mathrm{H} & 0.088006000 & -1.183132000 & -2.877179000 \\ \mathrm{H} & 2.123768000 & -2.044512000 & -1.258918000 \\ \mathrm{H} & 1.051732000 & -2.758977000 & 1.104677000 \\ \mathrm{H} & -1.626437000 & -2.337468000 & 0.981534000 \\ \mathrm{H} & -2.212386000 & -1.401369000 & -1.493567000 \\ \mathrm{Cl} & 2.481804000 & 1.116613000 & -1.317148000 \\ \mathrm{C} & -1.798832000 & 1.099110000 & -0.386688000 \\ \mathrm{C} & -3.200472000 & 1.103115000 & -0.201080000 \\ \mathrm{C} & -3.961938000 & 2.029860000 & -0.941285000 \\ \mathrm{C} & -3.366576000 & 2.925568000 & -1.847070000 \\ \mathrm{C} & -1.978958000 & 2.926027000 & -2.030827000 \\ \mathrm{C} & -1.215623000 & 2.009262000 & -1.281118000 \\ \mathrm{H} & -5.049539000 & 2.050308000 & -0.807075000 \\ \mathrm{H} & -3.991032000 & 3.631569000 & -2.402903000 \\ \mathrm{H} & -1.498272000 & 3.626928000 & -2.719533000 \\ \mathrm{H} & -0.110131000 & 2.066852000 & -1.425991000 \\ \mathrm{C} & -3.882492000 & 0.137407000 & 0.744562000 \\ \mathrm{H} & -4.913296000 & 0.454342000 & 0.971111000 \\ \mathrm{H} & -3.945618000 & -0.878817000 & 0.310496000 \\ \mathrm{H} & -3.337774000 & 0.046351000 & 1.700464000 \\ \mathrm{C} & 2.642411000 & 0.761117000 & -3.133685000 \\ \mathrm{H} & 3.596224000 & 1.229229000 & -3.406997000 \\ \mathrm{H} & 2.668917000 & -0.330902000 & -3.228609000 \\ \mathrm{Cl} & 1.332669000 & 1.429613000 & -4.103018000\end{array}$




\begin{tabular}{|c|c|c|}
\hline \multicolumn{3}{|c|}{ Vibrational Frequencies $\left(\mathrm{cm}^{-1}\right)$} \\
\hline 28.1 & 820.2 & 1457.7 \\
\hline 30.1 & 823.7 & 1467.2 \\
\hline 37.6 & 825.7 & 1480.2 \\
\hline 42.2 & 827.9 & 1561.1 \\
\hline 51.9 & 872.7 & 1582.6 \\
\hline 57.8 & 881.0 & 2885.1 \\
\hline 69.4 & 882.5 & 2967.5 \\
\hline 78.7 & 887.5 & 3026.4 \\
\hline 105.5 & 896.4 & 3066.9 \\
\hline 108.5 & 897.2 & 3069.9 \\
\hline 116.8 & 952.4 & 3111.2 \\
\hline 137.6 & 973.0 & 3130.6 \\
\hline 143.0 & 993.0 & 3145.4 \\
\hline 156.8 & 1009.2 & 3156.7 \\
\hline 178.8 & 1011.8 & 3170.1 \\
\hline 213.9 & 1019.1 & 3171.2 \\
\hline 236.5 & 1024.2 & 3181.5 \\
\hline 244.7 & 1031.7 & 3184.4 \\
\hline 246.9 & 1059.5 & 3188.1 \\
\hline 272.4 & 1062.0 & 3190.1 \\
\hline 283.8 & 1064.5 & 3199.5 \\
\hline 302.4 & 1067.6 & 3203.3 \\
\hline 320.2 & 1070.4 & 3216.8 \\
\hline 339.5 & 1109.1 & 3217.1 \\
\hline 390.2 & 1120.5 & \\
\hline 412.5 & 1123.4 & \\
\hline 481.4 & 1155.8 & \\
\hline 522.2 & 1167.8 & \\
\hline 564.8 & 1195.6 & \\
\hline 571.7 & 1256.8 & \\
\hline 575.2 & 1257.9 & \\
\hline 576.9 & 1261.8 & \\
\hline 578.7 & 1270.8 & \\
\hline 628.4 & 1338.3 & \\
\hline 675.7 & 1368.1 & \\
\hline 694.5 & 1371.5 & \\
\hline 741.9 & 1375.8 & \\
\hline 781.2 & 1382.0 & \\
\hline 785.3 & 1394.8 & \\
\hline 792.6 & 1423.6 & \\
\hline 799.9 & 1426.2 & \\
\hline 803.6 & 1433.8 & \\
\hline 806.4 & 1436.3 & \\
\hline 813.3 & 1442.2 & \\
\hline 817.9 & 1447.1 & \\
\hline
\end{tabular}


$\mathrm{Cp}_{2} \mathrm{Zr}(o$-tolyl $)\left(\mathrm{ClCH}_{2} \mathrm{Cl}\right)^{+}$, exo isomer $\left(\mathbf{2} \mathbf{a} \cdot \mathbf{C H}_{2} \mathbf{C l}_{2}-\mathbf{e x o}\right)$

$\begin{array}{lrrr}\text { Coordinates } & & & \\ & \mathrm{x} \AA & \mathrm{y} \AA & \mathrm{z} \AA \\ \mathrm{Zr} & 0.030042000 & -0.001368000 & 0.076934000 \\ \mathrm{C} & 0.119884000 & 0.018763000 & 2.317270000 \\ \mathrm{C} & 0.732256000 & 0.103996000 & 3.593902000 \\ \mathrm{C} & -0.096441000 & 0.019803000 & 4.732904000 \\ \mathrm{C} & -1.488375000 & -0.143168000 & 4.638761000 \\ \mathrm{C} & -2.105707000 & -0.223300000 & 3.384145000 \\ \mathrm{C} & -1.268185000 & -0.139014000 & 2.258319000 \\ \mathrm{H} & 0.364227000 & 0.088453000 & 5.724731000 \\ \mathrm{H} & -2.093425000 & -0.203934000 & 5.548551000 \\ \mathrm{H} & -3.187880000 & -0.347153000 & 3.285394000 \\ \mathrm{H} & -1.816898000 & -0.208702000 & 1.272108000 \\ \mathrm{Cl} & 2.802055000 & 0.095514000 & 0.541359000 \\ \mathrm{C} & 3.942201000 & -0.313983000 & -0.854962000 \\ \mathrm{Cl} & 3.686558000 & 0.645340000 & -2.318305000 \\ \mathrm{H} & 4.941523000 & -0.111766000 & -0.449515000 \\ \mathrm{H} & 3.793050000 & -1.377671000 & -1.075712000 \\ \mathrm{C} & -1.386961000 & 2.110311000 & -0.047426000 \\ \mathrm{C} & -0.073765000 & 2.535103000 & 0.318131000 \\ \mathrm{C} & 0.783722000 & 2.289406000 & -0.798886000 \\ \mathrm{C} & 0.015530000 & 1.682858000 & -1.835272000 \\ \mathrm{C} & -1.333118000 & 1.566502000 & -1.367810000 \\ \mathrm{H} & -2.277611000 & 2.187278000 & 0.577934000 \\ \mathrm{H} & 0.209796000 & 3.000302000 & 1.261526000 \\ \mathrm{H} & 1.845132000 & 2.530264000 & -0.852878000 \\ \mathrm{H} & 0.386084000 & 1.386515000 & -2.816919000 \\ \mathrm{H} & -2.182530000 & 1.184972000 & -1.935612000 \\ \mathrm{C} & -0.660652000 & -1.539850000 & -1.852838000 \\ \mathrm{C} & 0.722831000 & -1.779005000 & -1.620891000 \\ \mathrm{C} & 0.848125000 & -2.419173000 & -0.336706000 \\ \mathrm{C} & -0.440998000 & -2.529468000 & 0.231557000 \\ \mathrm{C} & -1.382065000 & -1.953426000 & -0.691835000 \\ \mathrm{H} & -1.091941000 & -1.100261000 & -2.752197000 \\ \mathrm{H} & 1.526023000 & -1.584382000 & -2.333400000 \\ \mathrm{H} & 1.772765000 & -2.761857000 & 0.129608000 \\ \mathrm{H} & -0.676524000 & -2.952413000 & 1.208029000 \\ \mathrm{H} & -2.466789000 & -1.932479000 & -0.570028000 \\ \mathrm{C} & 2.223245000 & 0.285791000 & 3.777102000 \\ \mathrm{H} & 2.594880000 & 1.170342000 & 3.230165000 \\ \mathrm{H} & 2.480322000 & 0.420665000 & 4.840048000 \\ \mathrm{H} & 2.786368000 & -0.590748000 & 3.409883000 \\ & & & \end{array}$




\begin{tabular}{|c|c|c|}
\hline \multicolumn{3}{|c|}{ Vibrational Frequencies $\left(\mathrm{cm}^{-1}\right)$} \\
\hline 24.8 & 824.5 & 1464.6 \\
\hline 35.4 & 825.8 & 1468.7 \\
\hline 40.9 & 827.0 & 1488.0 \\
\hline 43.7 & 831.5 & 1552.4 \\
\hline 51.5 & 877.3 & 1596.7 \\
\hline 64.9 & 885.7 & 2726.7 \\
\hline 74.0 & 888.3 & 2978.1 \\
\hline 96.4 & 890.9 & 3036.8 \\
\hline 120.9 & 893.2 & 3066.7 \\
\hline 126.0 & 895.1 & 3068.0 \\
\hline 131.0 & 952.0 & 3114.4 \\
\hline 150.2 & 972.7 & 3131.8 \\
\hline 161.6 & 980.3 & 3146.1 \\
\hline 174.1 & 1007.4 & 3153.0 \\
\hline 187.5 & 1013.5 & 3174.4 \\
\hline 209.0 & 1017.4 & 3176.3 \\
\hline 223.0 & 1028.1 & 3180.5 \\
\hline 245.9 & 1031.4 & 3187.7 \\
\hline 253.5 & 1063.0 & 3188.7 \\
\hline 261.2 & 1065.9 & 3197.0 \\
\hline 285.1 & 1068.8 & 3199.9 \\
\hline 294.8 & 1071.6 & 3202.1 \\
\hline 309.2 & 1074.8 & 3206.7 \\
\hline 336.5 & 1109.3 & 3212.5 \\
\hline 394.7 & 1122.4 & \\
\hline 430.4 & 1124.2 & \\
\hline 470.4 & 1159.6 & \\
\hline 520.7 & 1171.7 & \\
\hline 567.1 & 1214.6 & \\
\hline 570.4 & 1255.9 & \\
\hline 574.3 & 1261.2 & \\
\hline 575.7 & 1271.5 & \\
\hline 589.8 & 1276.9 & \\
\hline 624.1 & 1335.4 & \\
\hline 673.1 & 1368.8 & \\
\hline 693.4 & 1372.2 & \\
\hline 731.2 & 1376.4 & \\
\hline 778.0 & 1377.7 & \\
\hline 792.5 & 1393.0 & \\
\hline 797.0 & 1424.7 & \\
\hline 801.4 & 1426.4 & \\
\hline 802.1 & 1434.6 & \\
\hline 814.9 & 1436.9 & \\
\hline 816.1 & 1441.3 & \\
\hline 821.1 & 1456.1 & \\
\hline
\end{tabular}


$\mathrm{Cp}_{2} \mathrm{Zr}(o$-tolyl $)\left(\mathrm{ClCH}_{2} \mathrm{Cl}\right)^{+}, \boldsymbol{\gamma}$-agostic isomer $\left(\mathbf{2} \mathbf{a} \cdot \mathbf{C H}_{2} \mathbf{C l}_{2}-\boldsymbol{\gamma}\right.$-endo $)$

$\begin{array}{lrrr}\text { Coordinates } & & \\ & \mathrm{x} \AA & \mathrm{y} \AA & \mathrm{z} \AA \\ \mathrm{Zr} & 0.016631000 & 0.220484000 & 0.139031000 \\ \mathrm{C} & 0.037333000 & -0.382958000 & 2.626427000 \\ \mathrm{C} & 1.387993000 & -0.147443000 & 2.245745000 \\ \mathrm{C} & 1.519976000 & 1.242458000 & 1.899711000 \\ \mathrm{C} & 0.246295000 & 1.854134000 & 2.067932000 \\ \mathrm{C} & -0.677313000 & 0.846815000 & 2.488339000 \\ \mathrm{H} & -0.384710000 & -1.331575000 & 2.953618000 \\ \mathrm{H} & 2.194875000 & -0.882183000 & 2.256430000 \\ \mathrm{H} & 2.440124000 & 1.753286000 & 1.609945000 \\ \mathrm{H} & 0.017538000 & 2.909564000 & 1.920803000 \\ \mathrm{H} & -1.732775000 & 0.999266000 & 2.716563000 \\ \mathrm{C} & -1.645817000 & 1.924737000 & -0.580873000 \\ \mathrm{C} & -0.364426000 & 2.442630000 & -0.981163000 \\ \mathrm{C} & 0.134098000 & 1.611935000 & -2.019085000 \\ \mathrm{C} & -0.817963000 & 0.568963000 & -2.252793000 \\ \mathrm{C} & -1.934516000 & 0.784592000 & -1.388951000 \\ \mathrm{H} & -2.310982000 & 2.370124000 & 0.160132000 \\ \mathrm{H} & 0.120965000 & 3.337344000 & -0.590900000 \\ \mathrm{H} & 1.083170000 & 1.741601000 & -2.539787000 \\ \mathrm{H} & -0.735795000 & -0.217288000 & -3.004862000 \\ \mathrm{H} & -2.841075000 & 0.184005000 & -1.353673000 \\ \mathrm{C} & -1.514875000 & -1.421544000 & 0.417582000 \\ \mathrm{C} & -1.003507000 & -2.635231000 & -0.109525000 \\ \mathrm{C} & -1.763099000 & -3.819417000 & -0.026385000 \\ \mathrm{C} & -3.028212000 & -3.815849000 & 0.574182000 \\ \mathrm{C} & -3.546522000 & -2.618818000 & 1.094718000 \\ \mathrm{C} & -2.792877000 & -1.437728000 & 1.013536000 \\ \mathrm{H} & -1.355104000 & -4.749520000 & -0.440741000 \\ \mathrm{H} & -4.536994000 & -2.605335000 & 1.561178000 \\ \mathrm{H} & -3.222867000 & -0.513731000 & 1.420583000 \\ \mathrm{Cl} & 2.806088000 & -0.065443000 & -0.689527000 \\ \mathrm{C} & 3.337185000 & -1.072890000 & -2.151692000 \\ \mathrm{H} & 4.419819000 & -0.904562000 & -2.206022000 \\ \mathrm{H} & 3.086394000 & -2.112452000 & -1.910230000 \\ \mathrm{Cl} & 2.574290000 & -0.600680000 & -3.670299000 \\ \mathrm{C} & 0.377759000 & -2.666242000 & -0.747174000 \\ \mathrm{H} & 1.195731000 & -2.594131000 & -0.000835000 \\ \mathrm{H} & 0.547753000 & -3.605603000 & -1.301154000 \\ \mathrm{H} & 0.511441000 & -1.862618000 & -1.509986000 \\ \mathrm{H} & -3.609798000 & -4.740922000 & 0.630898000\end{array}$




\begin{tabular}{|c|c|c|}
\hline \multicolumn{3}{|c|}{ Vibrational Frequencies $\left(\mathrm{cm}^{-1}\right)$} \\
\hline 19.2 & 826.6 & 1445.1 \\
\hline 32.5 & 827.3 & 1468.6 \\
\hline 37.5 & 833.8 & 1482.2 \\
\hline 50.3 & 849.4 & 1565.7 \\
\hline 58.2 & 879.8 & 1583.0 \\
\hline 59.0 & 883.5 & 2868.8 \\
\hline 65.8 & 890.0 & 2955.2 \\
\hline 90.2 & 894.4 & 3035.6 \\
\hline 92.8 & 897.4 & 3070.8 \\
\hline 108.9 & 916.5 & 3082.5 \\
\hline 124.8 & 951.8 & 3098.1 \\
\hline 139.7 & 959.4 & 3124.7 \\
\hline 151.0 & 998.6 & 3142.2 \\
\hline 159.7 & 1010.1 & 3157.5 \\
\hline 163.8 & 1011.6 & 3172.8 \\
\hline 217.6 & 1020.6 & 3181.3 \\
\hline 240.0 & 1022.5 & 3183.1 \\
\hline 249.2 & 1038.7 & 3184.1 \\
\hline 252.6 & 1043.3 & 3188.9 \\
\hline 271.1 & 1063.5 & 3193.4 \\
\hline 282.4 & 1066.6 & 3200.7 \\
\hline 302.6 & 1068.1 & 3203.8 \\
\hline 327.1 & 1072.4 & 3217.2 \\
\hline 342.1 & 1121.3 & 3221.8 \\
\hline 344.5 & 1121.7 & \\
\hline 428.1 & 1123.7 & \\
\hline 475.9 & 1157.9 & \\
\hline 523.9 & 1167.0 & \\
\hline 561.7 & 1191.3 & \\
\hline 568.2 & 1256.4 & \\
\hline 574.5 & 1258.0 & \\
\hline 575.9 & 1260.9 & \\
\hline 583.9 & 1272.3 & \\
\hline 638.6 & 1325.7 & \\
\hline 698.3 & 1369.7 & \\
\hline 736.4 & 1371.2 & \\
\hline 737.6 & 1378.2 & \\
\hline 779.5 & 1380.8 & \\
\hline 802.0 & 1390.9 & \\
\hline 803.4 & 1424.6 & \\
\hline 810.4 & 1427.7 & \\
\hline 812.9 & 1434.5 & \\
\hline 818.8 & 1436.1 & \\
\hline 821.3 & 1440.4 & \\
\hline 825.1 & 1443.8 & \\
\hline
\end{tabular}


$\mathrm{Cp}_{2} \mathrm{Zr}(p$-tolyl $)\left(\mathrm{ClCH}_{2} \mathrm{Cl}\right)^{+}$, endo isomer (K-endo)

$\begin{array}{lrrr}\text { Coordinates } & & & \\ & \mathrm{x} \AA & \mathrm{y} \AA & \mathrm{z} \AA \\ \mathrm{Zr} & -0.062955000 & 0.259860000 & 0.258922000 \\ \mathrm{C} & 0.060244000 & -0.307805000 & 2.775019000 \\ \mathrm{C} & 1.378388000 & 0.033730000 & 2.363201000 \\ \mathrm{C} & 1.380506000 & 1.411030000 & 1.958050000 \\ \mathrm{C} & 0.058199000 & 1.915961000 & 2.145826000 \\ \mathrm{C} & -0.762648000 & 0.849077000 & 2.628881000 \\ \mathrm{H} & -0.272386000 & -1.287694000 & 3.117457000 \\ \mathrm{H} & 2.246614000 & -0.627630000 & 2.394925000 \\ \mathrm{H} & 2.248882000 & 1.983354000 & 1.628487000 \\ \mathrm{H} & -0.265162000 & 2.943637000 & 1.980278000 \\ \mathrm{H} & -1.822221000 & 0.914338000 & 2.876158000 \\ \mathrm{C} & -1.428061000 & 2.124983000 & -0.717982000 \\ \mathrm{C} & -0.062431000 & 2.498481000 & -0.955408000 \\ \mathrm{C} & 0.490496000 & 1.564830000 & -1.868285000 \\ \mathrm{C} & -0.529261000 & 0.611366000 & -2.208316000 \\ \mathrm{C} & -1.723190000 & 0.982794000 & -1.523073000 \\ \mathrm{H} & -2.137504000 & 2.659430000 & -0.084590000 \\ \mathrm{H} & 0.457530000 & 3.352133000 & -0.520237000 \\ \mathrm{H} & 1.511709000 & 1.590873000 & -2.252308000 \\ \mathrm{H} & -0.429946000 & -0.218786000 & -2.909852000 \\ \mathrm{H} & -2.685649000 & 0.479327000 & -1.592960000 \\ \mathrm{C} & -1.782485000 & -1.090980000 & 0.508401000 \\ \mathrm{C} & -1.121186000 & -2.179662000 & -0.083976000 \\ \mathrm{C} & -1.659910000 & -3.482252000 & -0.062818000 \\ \mathrm{C} & -2.911661000 & -3.710525000 & 0.534032000 \\ \mathrm{C} & -3.588351000 & -2.608286000 & 1.109996000 \\ \mathrm{C} & -3.035475000 & -1.321070000 & 1.105286000 \\ \mathrm{H} & -0.154191000 & -2.060703000 & -0.623689000 \\ \mathrm{H} & -1.115646000 & -4.310743000 & -0.528669000 \\ \mathrm{H} & -4.566259000 & -2.770761000 & 1.578113000 \\ \mathrm{H} & -3.590484000 & -0.499913000 & 1.573896000 \\ \mathrm{Cl} & 2.443939000 & -0.897653000 & -0.432961000 \\ \mathrm{C} & 2.776163000 & -1.376445000 & -2.193929000 \\ \mathrm{H} & 2.673989000 & -0.460120000 & -2.786927000 \\ \mathrm{H} & 3.807648000 & -1.749497000 & -2.173307000 \\ \mathrm{Cl} & 1.690789000 & -2.623819000 & -2.805752000 \\ \mathrm{C} & -3.530969000 & -5.089469000 & 0.552922000 \\ \mathrm{H} & -2.858234000 & -5.844098000 & 0.115239000 \\ \mathrm{H} & -3.771501000 & -5.405599000 & 1.583276000 \\ \mathrm{H} & -4.476600000 & -5.108410000 & -0.017944000 \\ & & & \end{array}$




\begin{tabular}{|c|c|c|}
\hline \multicolumn{3}{|c|}{ Vibrational Frequencies $\left(\mathrm{cm}^{-1}\right)$} \\
\hline 21.7 & 822.5 & 1465.9 \\
\hline 30.2 & 823.7 & 1470.9 \\
\hline 31.8 & 826.1 & 1487.7 \\
\hline 38.0 & 827.3 & 1560.4 \\
\hline 43.7 & 872.8 & 1581.2 \\
\hline 45.2 & 878.0 & 2914.0 \\
\hline 56.6 & 880.1 & 2982.3 \\
\hline 68.8 & 887.0 & 3041.1 \\
\hline 80.5 & 893.0 & 3069.6 \\
\hline 101.2 & 894.2 & 3071.4 \\
\hline 104.5 & 942.4 & 3097.8 \\
\hline 130.6 & 981.1 & 3116.5 \\
\hline 144.1 & 991.7 & 3118.9 \\
\hline 148.1 & 1009.2 & 3156.5 \\
\hline 171.4 & 1012.9 & 3170.8 \\
\hline 232.2 & 1018.4 & 3171.1 \\
\hline 245.2 & 1023.1 & 3181.1 \\
\hline 254.3 & 1028.0 & 3182.8 \\
\hline 262.4 & 1035.1 & 3186.5 \\
\hline 276.5 & 1063.7 & 3191.8 \\
\hline 284.2 & 1065.4 & 3197.7 \\
\hline 302.0 & 1069.1 & 3199.5 \\
\hline 325.0 & 1069.8 & 3208.0 \\
\hline 339.5 & 1121.6 & 3217.8 \\
\hline 346.4 & 1122.1 & \\
\hline 370.5 & 1123.2 & \\
\hline 478.9 & 1154.8 & \\
\hline 554.0 & 1188.2 & \\
\hline 565.0 & 1210.1 & \\
\hline 567.9 & 1255.1 & \\
\hline 573.6 & 1260.1 & \\
\hline 579.3 & 1269.6 & \\
\hline 581.4 & 1293.7 & \\
\hline 618.5 & 1336.6 & \\
\hline 687.1 & 1368.8 & \\
\hline 726.9 & 1372.4 & \\
\hline 739.9 & 1377.1 & \\
\hline 784.3 & 1378.7 & \\
\hline 788.7 & 1380.8 & \\
\hline 793.4 & 1395.7 & \\
\hline 803.0 & 1423.2 & \\
\hline 804.7 & 1434.8 & \\
\hline 811.5 & 1437.7 & \\
\hline 813.4 & 1441.5 & \\
\hline 821.7 & 1448.6 & \\
\hline
\end{tabular}


$\mathrm{Cp}_{2} \mathrm{Zr}\left(p\right.$-tolyl) $\left(\mathrm{ClCH}_{2} \mathrm{Cl}\right)^{+}$, exo isomer (K-exo)

$\begin{array}{lrrr}\text { Coordinates } & & & \\ & \mathrm{x} \AA & \mathrm{y} \AA & \mathrm{z} \AA \\ \mathrm{Zr} & -0.582726000 & -0.098172000 & -0.080500000 \\ \mathrm{C} & -0.167782000 & -0.112608000 & 2.102572000 \\ \mathrm{C} & 0.667499000 & -0.109983000 & 3.235780000 \\ \mathrm{C} & 0.111367000 & -0.008320000 & 4.518488000 \\ \mathrm{C} & -1.284785000 & 0.098231000 & 4.719585000 \\ \mathrm{C} & -2.124939000 & 0.100055000 & 3.589920000 \\ \mathrm{C} & -1.546864000 & -0.009073000 & 2.312416000 \\ \mathrm{H} & 1.755835000 & -0.183882000 & 3.132740000 \\ \mathrm{H} & 0.774073000 & -0.008971000 & 5.391577000 \\ \mathrm{H} & -3.210273000 & 0.182225000 & 3.710078000 \\ \mathrm{H} & -2.279231000 & -0.007561000 & 1.455551000 \\ \mathrm{Cl} & 2.193849000 & -0.184913000 & 0.237244000 \\ \mathrm{C} & 3.336252000 & 0.200269000 & -1.164741000 \\ \mathrm{H} & 3.269820000 & 1.282351000 & -1.329028000 \\ \mathrm{H} & 4.325105000 & -0.103344000 & -0.798820000 \\ \mathrm{Cl} & 2.962551000 & -0.658322000 & -2.664793000 \\ \mathrm{C} & -1.192093000 & 2.390762000 & 0.157522000 \\ \mathrm{C} & 0.100717000 & 2.360461000 & -0.412734000 \\ \mathrm{C} & -0.000420000 & 1.760362000 & -1.719415000 \\ \mathrm{C} & -1.369674000 & 1.455318000 & -1.954303000 \\ \mathrm{C} & -2.106114000 & 1.789884000 & -0.775739000 \\ \mathrm{H} & -1.443552000 & 2.765537000 & 1.149800000 \\ \mathrm{H} & 1.010614000 & 2.730047000 & 0.061376000 \\ \mathrm{H} & 0.809322000 & 1.630340000 & -2.439344000 \\ \mathrm{H} & -1.781703000 & 1.019207000 & -2.864365000 \\ \mathrm{H} & -3.186976000 & 1.703347000 & -0.648728000 \\ \mathrm{C} & -2.076431000 & -1.670104000 & -1.393651000 \\ \mathrm{C} & -0.779257000 & -1.775767000 & -1.990380000 \\ \mathrm{C} & 0.091653000 & -2.378542000 & -1.035061000 \\ \mathrm{C} & -0.650726000 & -2.632124000 & 0.159423000 \\ \mathrm{C} & -1.996560000 & -2.213657000 & -0.074711000 \\ \mathrm{H} & -2.979322000 & -1.292853000 & -1.875603000 \\ \mathrm{H} & -0.510909000 & -1.481144000 & -3.005272000 \\ \mathrm{H} & 1.144910000 & -2.610738000 & -1.192823000 \\ \mathrm{H} & -0.271416000 & -3.095589000 & 1.069426000 \\ \mathrm{H} & -2.822824000 & -2.301523000 & 0.632552000 \\ \mathrm{C} & -1.856631000 & 0.205184000 & 6.114654000 \\ \mathrm{H} & -1.424999000 & 1.064880000 & 6.656909000 \\ \mathrm{H} & -2.951152000 & 0.328550000 & 6.099218000 \\ \mathrm{H} & -1.626291000 & -0.697742000 & 6.707920000 \\ & & & \end{array}$




\begin{tabular}{|c|c|c|}
\hline \multicolumn{3}{|c|}{ Vibrational Frequencies $\left(\mathrm{cm}^{-1}\right)$} \\
\hline 25.1 & 824.7 & 1464.4 \\
\hline 27.9 & 825.1 & 1469.6 \\
\hline 28.3 & 826.0 & 1495.1 \\
\hline 38.4 & 828.5 & 1564.8 \\
\hline 47.8 & 878.9 & 1579.4 \\
\hline 53.5 & 880.7 & 2763.5 \\
\hline 61.8 & 884.4 & 2983.1 \\
\hline 67.8 & 889.5 & 3042.4 \\
\hline 84.9 & 894.7 & 3068.0 \\
\hline 113.5 & 897.2 & 3072.4 \\
\hline 121.1 & 942.2 & 3103.8 \\
\hline 122.6 & 976.4 & 3119.7 \\
\hline 147.6 & 990.8 & 3122.2 \\
\hline 155.1 & 1005.1 & 3153.5 \\
\hline 171.1 & 1014.3 & 3173.8 \\
\hline 228.8 & 1017.3 & 3175.6 \\
\hline 236.9 & 1018.5 & 3177.4 \\
\hline 251.0 & 1026.6 & 3185.4 \\
\hline 252.7 & 1033.7 & 3189.2 \\
\hline 263.4 & 1064.7 & 3194.3 \\
\hline 286.1 & 1065.9 & 3198.1 \\
\hline 293.7 & 1068.3 & 3200.3 \\
\hline 313.2 & 1069.2 & 3205.4 \\
\hline 340.1 & 1121.7 & 3211.2 \\
\hline 348.1 & 1123.0 & \\
\hline 376.8 & 1129.0 & \\
\hline 472.4 & 1156.0 & \\
\hline 547.7 & 1192.6 & \\
\hline 566.3 & 1218.9 & \\
\hline 570.8 & 1256.6 & \\
\hline 573.5 & 1258.9 & \\
\hline 575.9 & 1270.8 & \\
\hline 590.1 & 1300.0 & \\
\hline 619.3 & 1340.5 & \\
\hline 679.3 & 1369.0 & \\
\hline 713.9 & 1370.7 & \\
\hline 732.6 & 1375.0 & \\
\hline 782.4 & 1375.8 & \\
\hline 792.6 & 1377.7 & \\
\hline 795.8 & 1394.0 & \\
\hline 798.5 & 1427.8 & \\
\hline 804.6 & 1433.0 & \\
\hline 809.1 & 1438.6 & \\
\hline 810.7 & 1440.4 & \\
\hline 821.6 & 1454.5 & \\
\hline
\end{tabular}




$$
\mathrm{Cp}_{2} \mathrm{Zr}(\mathrm{Ph})(\mathrm{THF})^{+} \text {(D) }
$$

$\begin{array}{lrrr}\text { Coordinates } & & \\ & \mathrm{x} \AA & \mathrm{y} \AA & \mathrm{z} \AA \\ \mathrm{Zr} & -0.400988000 & -0.120979000 & -0.465225000 \\ \mathrm{O} & -0.112936000 & -0.171483000 & 1.794692000 \\ \mathrm{C} & 1.781004000 & 0.198742000 & -0.976280000 \\ \mathrm{C} & 2.599106000 & -0.626173000 & -0.152512000 \\ \mathrm{C} & 3.987989000 & -0.437532000 & -0.044329000 \\ \mathrm{C} & 4.617366000 & 0.554356000 & -0.810664000 \\ \mathrm{C} & 3.847550000 & 1.357658000 & -1.667625000 \\ \mathrm{C} & 2.457016000 & 1.184153000 & -1.739351000 \\ \mathrm{H} & 2.157978000 & -1.449819000 & 0.426139000 \\ \mathrm{H} & 4.579064000 & -1.086398000 & 0.611063000 \\ \mathrm{H} & 5.701960000 & 0.689826000 & -0.753106000 \\ \mathrm{H} & 4.332008000 & 2.126350000 & -2.278905000 \\ \mathrm{H} & 1.898186000 & 1.839901000 & -2.414897000 \\ \mathrm{C} & -0.782661000 & -0.991051000 & 2.836348000 \\ \mathrm{H} & -1.840519000 & -0.680341000 & 2.868385000 \\ \mathrm{H} & -0.709771000 & -2.041578000 & 2.521164000 \\ \mathrm{C} & 0.816606000 & 0.791510000 & 2.450630000 \\ \mathrm{H} & 0.695547000 & 1.758137000 & 1.940184000 \\ \mathrm{H} & 1.837778000 & 0.415135000 & 2.277717000 \\ \mathrm{C} & -0.040403000 & -0.672322000 & 4.133024000 \\ \mathrm{H} & -0.690989000 & -0.796739000 & 5.012757000 \\ \mathrm{H} & 0.833146000 & -1.334951000 & 4.255263000 \\ \mathrm{C} & 0.407255000 & 0.786154000 & 3.918832000 \\ \mathrm{H} & -0.428741000 & 1.484337000 & 4.097659000 \\ \mathrm{H} & 1.243475000 & 1.077003000 & 4.573727000 \\ \mathrm{C} & -1.553879000 & -2.397330000 & -0.186385000 \\ \mathrm{C} & -2.018135000 & -1.854332000 & -1.414797000 \\ \mathrm{C} & -0.903116000 & -1.769259000 & -2.309115000 \\ \mathrm{C} & 0.243523000 & -2.297637000 & -1.642133000 \\ \mathrm{C} & -0.153246000 & -2.667792000 & -0.323658000 \\ \mathrm{H} & -2.172792000 & -2.604704000 & 0.687962000 \\ \mathrm{H} & -3.044935000 & -1.561481000 & -1.634831000 \\ \mathrm{H} & -0.930712000 & -1.408785000 & -3.338509000 \\ \mathrm{H} & 1.240442000 & -2.395820000 & -2.067740000 \\ \mathrm{H} & 0.486934000 & -3.126419000 & 0.431759000 \\ \mathrm{C} & -1.434547000 & 2.131187000 & 0.180416000 \\ \mathrm{C} & -0.393733000 & 2.441572000 & -0.739034000 \\ \mathrm{C} & -0.748206000 & 1.882011000 & -2.007344000 \\ \mathrm{C} & -2.012286000 & 1.241306000 & -1.869863000 \\ \mathrm{C} & -2.428961000 & 1.367806000 & -0.507820000 \\ \mathrm{H} & -1.480229000 & 2.430783000 & 1.228440000 \\ \mathrm{H} & 0.512049000 & 3.008631000 & -0.524911000 \\ & & & \\ & & & \end{array}$




$\begin{array}{llll}\mathrm{H} & -0.176393000 & 1.963664000 & -2.931832000 \\ \mathrm{H} & -2.571974000 & 0.756980000 & -2.669713000 \\ \mathrm{H} & -3.366479000 & 1.001780000 & -0.085823000\end{array}$

Vibrational Frequencies $\left(\mathrm{cm}^{-1}\right)$

$\begin{array}{rrrr}25.2 & 818.0 & 1258.3 & 3186.1 \\ 43.6 & 823.5 & 1260.2 & 3190.2 \\ 52.8 & 824.6 & 1284.7 & 3197.3 \\ 58.8 & 826.9 & 1291.6 & 3199.4 \\ 70.9 & 827.5 & 1327.7 & 3206.4 \\ 76.0 & 829.9 & 1336.8 & 3218.7 \\ 85.5 & 831.2 & 1339.5 & \\ 119.6 & 838.8 & 1354.4 & \\ 122.9 & 879.0 & 1371.4 & \\ 132.2 & 881.7 & 1376.6 & \\ 148.2 & 882.8 & 1380.8 & \\ 154.1 & 888.9 & 1384.2 & \\ 179.9 & 900.8 & 1417.8 & \\ 193.6 & 902.3 & 1437.8 & \\ 208.1 & 916.6 & 1440.5 & \\ 228.7 & 940.1 & 1445.3 & \\ 243.7 & 947.2 & 1447.2 & \\ 252.7 & 972.0 & 1467.3 & \\ 258.3 & 978.3 & 1468.9 & \\ 265.1 & 981.5 & 1473.4 & \\ 293.3 & 1002.4 & 1490.1 & \\ 325.1 & 1013.9 & 1502.4 & \\ 345.5 & 1017.3 & 1564.4 & \\ 383.1 & 1023.3 & 1572.8 & \\ 442.8 & 1025.1 & 3006.8 & \\ 557.2 & 1035.7 & 3007.5 & \\ 565.1 & 1047.5 & 3016.1 & \\ 568.6 & 1067.0 & 3023.6 & \\ 571.8 & 1067.6 & 3066.2 & \\ 575.4 & 1071.5 & 3069.1 & \\ 606.2 & 1072.9 & 3073.5 & \\ 633.5 & 1079.6 & 3086.1 & \\ 666.1 & 1124.6 & 3088.9 & \\ 689.7 & 1126.4 & 3109.1 & \\ 719.0 & 1130.5 & 3116.9 & \\ 794.2 & 1153.6 & 3126.3 & \\ 798.1 & 1160.3 & 3140.9 & \\ 801.6 & 1165.5 & 3171.8 & \\ 807.4 & 1193.0 & 3172.9 & \\ 810.2 & 1225.8 & 3179.7 & \\ 814.9 & 1238.3 & 3182.4 & \\ & & & \\ & & \end{array}$




\section{$\mathrm{Cp}_{2} \mathrm{Hf}(\mathrm{Ph})\left(\mathrm{ClC}_{6} \mathrm{H}_{5}\right)^{+}\left(\mathbf{2 g} \cdot \mathbf{C}_{\mathbf{6}} \mathbf{H}_{5} \mathbf{C l}\right)$}

\begin{tabular}{|c|c|c|c|}
\hline \multicolumn{4}{|c|}{ Coordinates } \\
\hline & $\mathrm{x} \AA$ & y $\AA$ & $\mathrm{z} \AA \AA$ \\
\hline $\mathrm{C}$ & 0.306459000 & -0.777703000 & 0.164319000 \\
\hline $\mathrm{C}$ & 0.357654000 & -0.337219000 & 1.530381000 \\
\hline $\mathrm{C}$ & 1.721547000 & -0.119388000 & 1.876992000 \\
\hline $\mathrm{C}$ & 2.515063000 & -0.394191000 & 0.716936000 \\
\hline $\mathrm{C}$ & 1.637154000 & -0.828001000 & -0.330622000 \\
\hline $\mathrm{C}$ & 1.632781000 & 1.895442000 & -2.329000000 \\
\hline $\mathrm{C}$ & 1.095603000 & 3.149338000 & -1.895048000 \\
\hline $\mathrm{C}$ & 2.025368000 & 3.745411000 & -0.994382000 \\
\hline $\mathrm{C}$ & 3.142899000 & 2.861537000 & -0.868873000 \\
\hline $\mathrm{C}$ & 2.907446000 & 1.729059000 & -1.706301000 \\
\hline $\mathrm{C}$ & -2.021099000 & 3.616693000 & -0.451900000 \\
\hline $\mathrm{C}$ & -1.720837000 & 4.622647000 & 0.468306000 \\
\hline $\mathrm{C}$ & -2.182547000 & 5.917649000 & 0.174002000 \\
\hline $\mathrm{C}$ & 22000 & 6.165642000 & -0.995880000 \\
\hline $\mathrm{C}$ & -3.203919000 & 5.120392000 & -1.888254000 \\
\hline $\mathrm{C}$ & -2.754531000 & 3.814947000 & -1.624330000 \\
\hline $\mathrm{Cl}$ & -1.490219000 & 1.919077000 & -0.086005000 \\
\hline $\mathrm{Hf}$ & 1.189050000 & 1.626226000 & 0.125276000 \\
\hline $\mathrm{H}$ & -0.592708000 & -1.063149000 & 5000 \\
\hline $\mathrm{H}$ & 33000 & & \\
\hline $\mathrm{H}$ & 2.096931000 & 0.202040000 & 2.8471 \\
\hline $\mathrm{H}$ & 3.604046000 & -0.346817000 & 0.664013000 \\
\hline $\mathrm{H}$ & 37000 & 9029000 & -1.3322 \\
\hline $\mathrm{H}$ & 1.168290000 & 1.206132000 & -3.036353000 \\
\hline $\mathrm{H}$ & 96000 & 3.582068000 & -2.206913000 \\
\hline $\mathrm{H}$ & 1.904896000 & 4.702838000 & -0.489389000 \\
\hline $\mathrm{H}$ & 4.036017000 & 3.029867000 & -0.265552000 \\
\hline $\mathrm{H}$ & 3.591819000 & 0.893782000 & -1.855746000 \\
\hline $\mathrm{H}$ & -1.154357000 & & 1.379436000 \\
\hline $\mathrm{H}$ & -1.967750000 & 6.729646000 & 0.875042000 \\
\hline $\mathrm{H}$ & -3.274644000 & 7.176850000 & -1.210415000 \\
\hline $\mathrm{H}$ & -3.784110000 & 5.309972000 & -2.796046000 \\
\hline $\mathrm{H}$ & -2.981073000 & 2.988588000 & -2.302919000 \\
\hline $\mathrm{C}$ & 03000 & 2.905348000 & 1.9609 \\
\hline $\mathrm{C}$ & 0.471962000 & 2.761901000 & 2.939070000 \\
\hline $\mathrm{C}$ & 388000 & 3.619647000 & 4.046152000 \\
\hline $\mathrm{C}$ & 1.311121000 & 4.627162000 & 4.250113000 \\
\hline $\mathrm{C}$ & 2.364670000 & 4.775563000 & 3.333070000 \\
\hline $\mathrm{C}$ & 2.444575000 & 3.938734000 & 2.210299000 \\
\hline $\mathrm{H}$ & -0.274120000 & 1.961297000 & 2.847870000 \\
\hline $\mathrm{H}$ & -0.461707000 & 3.480976000 & 4.761763000 \\
\hline $\mathrm{H}$ & 1.244215000 & 5.281415000 & 5.125128000 \\
\hline
\end{tabular}




$\begin{array}{llll}\mathrm{H} & 3.123713000 & 5.548980000 & 3.491543000 \\ \mathrm{H} & 3.279204000 & 4.100214000 & 1.519282000\end{array}$

Vibrational Frequencies $\left(\mathrm{cm}^{-1}\right)$

$\begin{array}{rrr}22.9 & 806.4 & 1255.9 \\ 34.7 & 806.9 & 1258.1 \\ 40.2 & 814.0 & 1284.2 \\ 49.3 & 816.7 & 1303.0 \\ 56.7 & 822.8 & 1338.7 \\ 63.6 & 826.3 & 1352.3 \\ 76.7 & 827.1 & 1368.0 \\ 81.9 & 827.9 & 1372.0 \\ 95.8 & 830.3 & 1377.3 \\ 111.6 & 833.6 & 1380.0 \\ 127.9 & 883.9 & 1417.5 \\ 139.7 & 887.2 & 1431.2 \\ 147.6 & 887.6 & 1438.5 \\ 181.1 & 893.6 & 1440.3 \\ 221.2 & 896.5 & 1445.1 \\ 225.0 & 901.7 & 1450.0 \\ 252.5 & 939.2 & 1466.4 \\ 261.4 & 941.0 & 1471.6 \\ 268.2 & 963.3 & 1563.6 \\ 280.4 & 972.3 & 1571.0 \\ 287.2 & 974.3 & 1576.3 \\ 302.2 & 979.2 & 1602.2 \\ 305.1 & 1007.5 & 3077.4 \\ 378.7 & 1010.5 & 3102.7 \\ 380.7 & 1013.2 & 3117.5 \\ 402.2 & 1015.5 & 3125.0 \\ 437.3 & 1021.1 & 3132.5 \\ 456.6 & 1021.8 & 3139.1 \\ 579.4 & 1046.6 & 3140.3 \\ 581.5 & 1054.2 & 3146.1 \\ 585.3 & 1065.8 & 3152.1 \\ 589.5 & 1066.4 & 3157.4 \\ 596.4 & 1069.9 & 3169.5 \\ 608.6 & 1071.8 & 3172.1 \\ 637.2 & 1079.5 & 3178.1 \\ 658.2 & 1079.6 & 3179.2 \\ 664.7 & 1119.5 & 3185.7 \\ 689.3 & 1123.0 & 3186.4 \\ 718.9 & 1165.4 & 3195.1 \\ 729.6 & 1167.5 & 3197.3 \\ 799.2 & 1174.5 & 3209.8 \\ 803.5 & 1198.4 & 3214.0\end{array}$


$\mathrm{Cp}_{2} \mathrm{Zr}(\mathrm{Ph})\left(\mathrm{ClC}_{6} \mathrm{H}_{5}\right)^{+}$, isomer a $\left(\mathbf{2} \mathbf{d} \cdot \mathbf{C}_{6} \mathbf{H}_{5} \mathbf{C l}\right.$-endo(a) $)$

\begin{tabular}{|c|c|c|c|}
\hline \multicolumn{4}{|c|}{ Coordinates } \\
\hline & $\mathrm{x} \AA$ & $y=1$ & $\mathrm{z} \AA$ \\
\hline $\mathrm{C}$ & 0.257753000 & -0.718484000 & 0.273080000 \\
\hline $\mathrm{C}$ & 0.438421000 & -0.317847000 & 1.639276000 \\
\hline $\mathrm{C}$ & 1.832945000 & -0.133283000 & 1.868255000 \\
\hline $\mathrm{C}$ & 2.511714000 & -0.378025000 & 0.633544000 \\
\hline $\mathrm{C}$ & 1.535328000 & -0.762045000 & -0.345615000 \\
\hline $\mathrm{C}$ & 1.536739000 & 2.110012000 & -2.215461000 \\
\hline $\mathrm{C}$ & 1.118190000 & 3.371336000 & -1.678332000 \\
\hline $\mathrm{C}$ & 2.128774000 & 3.833886000 & -0.789933000 \\
\hline $\mathrm{C}$ & 3.176652000 & 2.862637000 & -0.769682000 \\
\hline $\mathrm{C}$ & 2.822406000 & 1.812664000 & -1.669963000 \\
\hline $\mathrm{C}$ & -2.156849000 & 3.563400000 & -0.562168000 \\
\hline $\mathrm{C}$ & -1.920894000 & 4.604523000 & 0.339063000 \\
\hline $\mathrm{C}$ & -2.413358000 & 6670000 & 0.000663000 \\
\hline $\mathrm{C}$ & -3.118966000 & 3621000 & -1.196526000 \\
\hline $\mathrm{C}$ & -3.340771000 & 8683000 & -2.071506000 \\
\hline $\mathrm{C}$ & -2.856929000 & 6055000 & -1.762146000 \\
\hline $\mathrm{Cl}$ & -1.578850000 & 1.899479000 & -0.150011000 \\
\hline $\mathrm{Zr}$ & 1.164128000 & 1.684581000 & 0.218402000 \\
\hline $\mathrm{H}$ & -0.691776000 & -0.972754000 & -0.201966000 \\
\hline $\mathrm{H}$ & 195000 & & \\
\hline $\mathrm{H}$ & 2.301025000 & 641000 & 2.81231 \\
\hline $\mathrm{H}$ & 3.591626000 & -0.344775000 & 0.483417000 \\
\hline $\mathrm{H}$ & 617000 & 856000 & -1.3788 \\
\hline $\mathrm{H}$ & 0.994112000 & 1.510557000 & -2.948107000 \\
\hline $\mathrm{H}$ & 3816000 & 0580000 & -1.915935000 \\
\hline $\mathrm{H}$ & 2.103101000 & 4.757009000 & -0.211800000 \\
\hline $\mathrm{H}$ & 4.102526000 & 2.928559000 & -0.197978000 \\
\hline $\mathrm{H}$ & 3.435804000 & 0.944579000 & -1.910904000 \\
\hline $\mathrm{H}$ & 1986000 & & 1.271955000 \\
\hline $\mathrm{H}$ & -2.245217000 & 6.711787000 & 0.687129000 \\
\hline $\mathrm{H}$ & -3.500816000 & 7.067368000 & -1.446963000 \\
\hline $\mathrm{H}$ & 92000 & 5.147909000 & -3.002599000 \\
\hline $\mathrm{H}$ & -3.029993000 & 2.868949000 & -2.430559000 \\
\hline $\mathrm{C}$ & 1.777397000 & 2.814487000 & 2.040704000 \\
\hline $\mathrm{C}$ & 0.548545000 & 2.889321000 & 2.741403000 \\
\hline $\mathrm{C}$ & $0.354 \mathrm{C}$ & 3.792698000 & 3.806884000 \\
\hline $\mathrm{C}$ & 1.423372000 & 4.595545000 & 4.227254000 \\
\hline $\mathrm{C}$ & 2.667931000 & 4.502457000 & 3.577398000 \\
\hline $\mathrm{C}$ & 2.837776000 & 3.629245000 & 2.492734000 \\
\hline $\mathrm{H}$ & -0.290583000 & 2.214042000 & 2.498968000 \\
\hline $\mathrm{H}$ & -0.607237000 & 3.830446000 & 4.328539000 \\
\hline $\mathrm{H}$ & 1.295416000 & 5.278527000 & 5.072751000 \\
\hline
\end{tabular}




$\begin{array}{llll}\mathrm{H} & 3.505942000 & 5.121512000 & 3.914490000 \\ \mathrm{H} & 3.814342000 & 3.591880000 & 1.996300000\end{array}$

Vibrational Frequencies $\left(\mathrm{cm}^{-1}\right)$

$\begin{array}{ccc}22.4 & 804.0 & 1256.3 \\ 31.8 & 806.5 & 1258.3 \\ 34.3 & 812.5 & 1291.0 \\ 38.0 & 812.9 & 1302.2 \\ 41.1 & 819.9 & 1341.4 \\ 54.1 & 822.7 & 1352.4 \\ 58.5 & 824.0 & 1368.3 \\ 78.4 & 824.6 & 1374.8 \\ 90.0 & 826.4 & 1379.0 \\ 104.2 & 827.9 & 1381.4 \\ 121.8 & 880.7 & 1416.1 \\ 143.0 & 881.7 & 1431.4 \\ 160.7 & 887.4 & 1439.6 \\ 185.4 & 889.7 & 1443.6 \\ 214.3 & 891.9 & 1446.3 \\ 239.6 & 892.4 & 1449.9 \\ 251.6 & 934.4 & 1468.5 \\ 263.2 & 939.3 & 1472.2 \\ 268.8 & 967.0 & 1564.0 \\ 287.2 & 970.3 & 1572.0 \\ 307.3 & 973.2 & 1574.2 \\ 330.5 & 973.8 & 1602.0 \\ 349.4 & 1005.7 & 3013.7 \\ 377.2 & 1008.8 & 3101.0 \\ 383.6 & 1014.6 & 3118.7 \\ 402.0 & 1015.2 & 3127.3 \\ 449.4 & 1019.6 & 3131.6 \\ 457.8 & 1021.8 & 3138.2 \\ 567.0 & 1040.7 & 3142.6 \\ 568.9 & 1049.5 & 3144.8 \\ 574.0 & 1062.8 & 3152.4 \\ 579.0 & 1065.5 & 3158.0 \\ 597.8 & 1069.0 & 3172.0 \\ 601.3 & 1069.7 & 3176.2 \\ 636.2 & 1077.9 & 3181.7 \\ 662.9 & 1079.9 & 3184.8 \\ 666.6 & 1121.0 & 3187.6 \\ 686.5 & 1124.7 & 3191.9 \\ 712.9 & 1165.5 & 3197.6 \\ 729.3 & 1167.6 & 3200.8 \\ 797.0 & 1174.8 & 3209.6 \\ 800.6 & 1185.7 & 3217.6\end{array}$


$\mathrm{Cp}_{2} \mathrm{Zr}(\mathrm{Ph})\left(\mathrm{ClC}_{6} \mathrm{H}_{5}\right)^{+}$, isomer b $\left(\mathbf{2 d} \cdot \mathbf{C}_{6} \mathbf{H}_{5} \mathbf{C l}\right.$-endo(b) $)$

$\begin{array}{lrrr}\text { Coordinates } & & & \\ & \mathrm{x} \AA & \mathrm{y} \AA & \mathrm{z} \AA \\ \mathrm{C} & -4.530710000 & 1.428814000 & -0.517602000 \\ \mathrm{C} & -5.292421000 & 0.653600000 & 0.370312000 \\ \mathrm{C} & -4.869575000 & -0.637483000 & 0.723423000 \\ \mathrm{C} & -3.677740000 & -1.165355000 & 0.198829000 \\ \mathrm{C} & -2.937834000 & -0.358432000 & -0.670714000 \\ \mathrm{C} & -3.334179000 & 0.925971000 & -1.056194000 \\ \mathrm{Cl} & -1.435515000 & -1.032607000 & -1.406312000 \\ \mathrm{Zr} & 0.952814000 & -0.568483000 & 0.031310000 \\ \mathrm{C} & 1.503978000 & 1.550970000 & 0.352484000 \\ \mathrm{C} & 2.544437000 & 2.467073000 & 0.591118000 \\ \mathrm{C} & 2.324341000 & 3.845454000 & 0.441597000 \\ \mathrm{C} & 1.064141000 & 4.328173000 & 0.042611000 \\ \mathrm{C} & 0.011569000 & 3.433707000 & -0.189265000 \\ \mathrm{C} & 0.240487000 & 2.053330000 & -0.014472000 \\ \mathrm{C} & 3.153347000 & -0.327566000 & -1.205530000 \\ \mathrm{C} & 2.150893000 & -0.282670000 & -2.221448000 \\ \mathrm{C} & 1.520074000 & -1.560969000 & -2.275858000 \\ \mathrm{C} & 2.099466000 & -2.390372000 & -1.270091000 \\ \mathrm{C} & 3.119196000 & -1.625937000 & -0.612078000 \\ \mathrm{C} & 0.727142000 & -0.201452000 & 2.544121000 \\ \mathrm{C} & 1.756699000 & -1.170718000 & 2.347828000 \\ \mathrm{C} & 1.153194000 & -2.362429000 & 1.827483000 \\ \mathrm{C} & -0.233722000 & -2.111962000 & 1.656549000 \\ \mathrm{C} & -0.498847000 & -0.766408000 & 2.086932000 \\ \mathrm{H} & -0.966254000 & -2.824755000 & 1.275599000 \\ \mathrm{H} & 1.668475000 & -3.296949000 & 1.605124000 \\ \mathrm{H} & -1.476440000 & -0.281985000 & 2.108192000 \\ \mathrm{H} & 0.861864000 & 0.798551000 & 2.952667000 \\ \mathrm{H} & 2.808548000 & -1.045150000 & 2.608376000 \\ \mathrm{H} & 0.740784000 & -1.860426000 & -2.979335000 \\ \mathrm{H} & 1.919137000 & 0.576629000 & -2.852322000 \\ \mathrm{H} & 1.850781000 & -3.434707000 & -1.078691000 \\ \mathrm{H} & 3.787409000 & -1.990144000 & 0.168698000 \\ \mathrm{H} & 3.831381000 & 0.482740000 & -0.943468000 \\ \mathrm{H} & -0.640872000 & 1.386188000 & -0.139796000 \\ \mathrm{H} & 3.537515000 & 2.114000000 & 0.893331000 \\ \mathrm{H} & 3.141473000 & 4.549877000 & 0.628820000 \\ \mathrm{H} & -0.979516000 & 3.801119000 & -0.473684000 \\ \mathrm{H} & 0.901570000 & 5.403725000 & -0.075834000 \\ \mathrm{H} & -2.744096000 & 1.509261000 & -1.767959000 \\ \mathrm{H} & -3.352771000 & -2.178406000 & 0.447805000 \\ \mathrm{H} & -5.469744000 & -1.249336000 & 1.403293000 \\ & & & \end{array}$




$\begin{array}{lrrr}\mathrm{H} & -4.867372000 & 2.428716000 & -0.806830000 \\ \mathrm{H} & -6.224281000 & 1.052640000 & 0.780709000\end{array}$

Vibrational Frequencies $\left(\mathrm{cm}^{-1}\right)$

\begin{tabular}{|c|c|c|c|}
\hline 12.9 & 793.6 & 1164.4 & 3188.5 \\
\hline 19.9 & 799.8 & 1167.6 & 3191.9 \\
\hline 33.7 & 800.6 & 1172.7 & 3197.5 \\
\hline 38.2 & 807.1 & 1183.1 & 3200.5 \\
\hline 48.3 & 811.3 & 1255.2 & 3216.0 \\
\hline 55.6 & 815.1 & 1257.1 & 3216.2 \\
\hline 59.4 & 818.9 & 1294.3 & \\
\hline 81.3 & 821.6 & 1299.3 & \\
\hline 92.6 & 823.5 & 1344.7 & \\
\hline 108.6 & 826.1 & 1353.5 & \\
\hline 123.3 & 826.5 & 1369.2 & \\
\hline 135.3 & 831.6 & 1373.3 & \\
\hline 169.1 & 871.3 & 1377.0 & \\
\hline 189.5 & 882.6 & 1381.3 & \\
\hline 223.4 & 886.1 & 1417.5 & \\
\hline 239.3 & 891.9 & 1435.5 & \\
\hline 249.6 & 896.1 & 1438.3 & \\
\hline 257.9 & 901.2 & 1440.2 & \\
\hline 273.9 & 928.4 & 1446.3 & \\
\hline 279.8 & 935.9 & 1449.1 & \\
\hline 315.4 & 967.7 & 1469.0 & \\
\hline 322.3 & 969.7 & 1476.5 & \\
\hline 346.5 & 970.1 & 1566.8 & \\
\hline 373.9 & 973.4 & 1576.0 & \\
\hline 387.5 & 1006.5 & 1576.2 & \\
\hline 402.8 & 1008.5 & 1601.3 & \\
\hline 450.9 & 1012.8 & 2924.5 & \\
\hline 458.6 & 1015.1 & 3098.8 & \\
\hline 565.2 & 1018.9 & 3121.2 & \\
\hline 569.9 & 1021.0 & 3128.9 & \\
\hline 574.3 & 1035.0 & 3132.8 & \\
\hline 577.2 & 1051.1 & 3139.3 & \\
\hline 599.3 & 1062.0 & 3144.4 & \\
\hline 600.8 & 1064.2 & 3148.8 & \\
\hline 636.7 & 1066.5 & 3154.0 & \\
\hline 666.5 & 1069.8 & 3158.3 & \\
\hline 667.7 & 1078.2 & 3172.0 & \\
\hline 678.8 & 1082.9 & 3176.3 & \\
\hline 702.7 & 1122.2 & 3180.5 & \\
\hline 727.2 & 1123.2 & 3184.8 & \\
\hline
\end{tabular}


$\mathrm{Cp}_{2} \mathrm{Hf}(o$-tolyl $)\left(\mathrm{ClC}_{6} \mathrm{H}_{5}\right)^{+}$, endo isomer $\left(\mathbf{2} \mathbf{f} \cdot \mathbf{C}_{6} \mathbf{H}_{5} \mathbf{C l}\right.$-endo $)$

$\begin{array}{lrrr}\text { Coordinates } & & & \\ & \mathrm{x} \AA & \mathrm{y} \AA & \mathrm{z} \AA \\ \mathrm{C} & -0.824517000 & -2.245659000 & -1.990755000 \\ \mathrm{C} & -1.676063000 & -1.130319000 & -2.268316000 \\ \mathrm{C} & -2.719946000 & -1.117510000 & -1.296160000 \\ \mathrm{C} & -2.492572000 & -2.206240000 & -0.395681000 \\ \mathrm{C} & -1.320527000 & -2.909145000 & -0.833027000 \\ \mathrm{C} & 0.946832000 & -1.514945000 & 1.794609000 \\ \mathrm{C} & 0.867327000 & -0.092699000 & 1.975108000 \\ \mathrm{C} & -0.459392000 & 0.225041000 & 2.384117000 \\ \mathrm{C} & -1.213939000 & -0.987910000 & 2.414325000 \\ \mathrm{C} & -0.332420000 & -2.065561000 & 2.075900000 \\ \mathrm{C} & 3.216488000 & 0.116086000 & -0.567873000 \\ \mathrm{C} & 3.189641000 & 1.506202000 & -0.425762000 \\ \mathrm{C} & 4.306962000 & 2.119052000 & 0.166808000 \\ \mathrm{C} & 5.402991000 & 1.349016000 & 0.587269000 \\ \mathrm{C} & 5.395001000 & -0.044105000 & 0.418210000 \\ \mathrm{C} & 4.289683000 & -0.684440000 & -0.167837000 \\ \mathrm{Cl} & 1.824019000 & -0.712378000 & -1.371018000 \\ \mathrm{H} & -0.611343000 & -0.635413000 & 0.006441000 \\ \mathrm{H} & 0.038253000 & -2.557839000 & -2.582374000 \\ \mathrm{H} & -1.563684000 & -0.425580000 & -3.093470000 \\ \mathrm{H} & -3.545833000 & -0.409038000 & -1.255184000 \\ \mathrm{H} & -3.134940000 & -2.492020000 & 0.438254000 \\ \mathrm{H} & -0.904943000 & -3.812300000 & -0.385893000 \\ \mathrm{H} & 1.837227000 & -2.077095000 & 1.509434000 \\ \mathrm{H} & 1.688275000 & 0.615938000 & 1.856642000 \\ \mathrm{H} & -0.833710000 & 1.219908000 & 2.618605000 \\ \mathrm{H} & -2.261880000 & -1.082631000 & 2.700656000 \\ \mathrm{H} & -0.596878000 & -3.122673000 & 2.048448000 \\ \mathrm{H} & 2.333249000 & 2.097514000 & -0.759379000 \\ \mathrm{H} & 4.315119000 & 3.206150000 & 0.289424000 \\ \mathrm{H} & 6.269028000 & 1.837596000 & 1.042524000 \\ \mathrm{H} & 6.251260000 & -0.645466000 & 0.737352000 \\ \mathrm{H} & 4.277627000 & -1.766907000 & -0.319111000 \\ \mathrm{C} & -1.641806000 & 1.323814000 & -0.062245000 \\ \mathrm{C} & -0.743939000 & 1.889172000 & -0.991902000 \\ \mathrm{C} & -0.774205000 & 3.252096000 & -1.349905000 \\ \mathrm{C} & -1.761154000 & 4.061658000 & -0.775273000 \\ \mathrm{C} & -2.687595000 & 3.510142000 & 0.127103000 \\ \mathrm{C} & -2.645547000 & 2.151377000 & 0.498830000 \\ \mathrm{H} & 0.001445000 & 1.261495000 & -1.527635000 \\ \mathrm{H} & -0.070695000 & 3.657052000 & -2.084504000 \\ \mathrm{H} & -1.826624000 & 5.120433000 & -1.044109000 \\ & & & \end{array}$




$\begin{array}{llll}\mathrm{H} & -3.466387000 & 4.153613000 & 0.552611000 \\ \mathrm{C} & -3.657741000 & 1.609204000 & 1.487513000 \\ \mathrm{H} & -4.545531000 & 2.259138000 & 1.553524000 \\ \mathrm{H} & -3.997834000 & 0.596861000 & 1.208850000 \\ \mathrm{H} & -3.235543000 & 1.540041000 & 2.508541000\end{array}$

\begin{tabular}{|c|c|c|c|}
\hline \multicolumn{4}{|c|}{ Vibrational Frequencies $\left(\mathrm{cm}^{-1}\right)$} \\
\hline 19.8 & 692.8 & 1068.9 & 3121.5 \\
\hline 29.8 & 702.3 & 1079.9 & 3133.1 \\
\hline 41.3 & 727.7 & 1103.5 & 3139.8 \\
\hline 47.5 & 782.5 & 1120.0 & 3140.8 \\
\hline 50.6 & 797.9 & 1123.2 & 3149.5 \\
\hline 57.0 & 798.9 & 1164.5 & 3154.6 \\
\hline 65.3 & 804.8 & 1167.5 & 3158.1 \\
\hline 86.1 & 807.4 & 1174.5 & 3170.6 \\
\hline 96.6 & 810.2 & 1191.3 & 3175.3 \\
\hline 121.3 & 813.0 & 1251.5 & 3179.5 \\
\hline 131.3 & 819.9 & 1254.8 & 3182.0 \\
\hline 133.4 & 825.3 & 1259.8 & 3188.0 \\
\hline 142.9 & 826.8 & 1301.9 & 3188.8 \\
\hline 159.1 & 827.8 & 1333.3 & 3195.7 \\
\hline 194.3 & 829.1 & 1352.7 & 3199.5 \\
\hline 216.9 & 832.3 & 1368.6 & 3211.6 \\
\hline 238.3 & 883.5 & 1372.1 & 3215.5 \\
\hline 249.1 & 887.2 & 1376.8 & \\
\hline 251.7 & 888.0 & 1381.5 & \\
\hline 276.2 & 893.3 & 1395.7 & \\
\hline 287.0 & 896.2 & 1420.0 & \\
\hline 287.8 & 899.7 & 1433.1 & \\
\hline 297.4 & 936.9 & 1435.8 & \\
\hline 300.1 & 952.4 & 1441.3 & \\
\hline 383.1 & 968.0 & 1445.0 & \\
\hline 384.7 & 973.4 & 1449.5 & \\
\hline 400.9 & 973.9 & 1457.8 & \\
\hline 418.9 & 1001.1 & 1468.5 & \\
\hline 458.6 & 1008.1 & 1468.9 & \\
\hline 479.3 & 1010.1 & 1481.4 & \\
\hline 522.1 & 1014.7 & 1564.5 & \\
\hline 577.5 & 1018.2 & 1574.1 & \\
\hline 584.6 & 1021.5 & 1576.9 & \\
\hline 589.4 & 1034.9 & 1601.9 & \\
\hline 591.6 & 1049.9 & 2928.3 & \\
\hline 598.0 & 1055.1 & 2965.8 & \\
\hline 629.8 & 1061.1 & 3026.2 & \\
\hline 662.7 & 1064.2 & 3064.7 & \\
\hline 666.7 & 1065.1 & 3107.9 & \\
\hline
\end{tabular}


$\mathrm{Cp}_{2} \mathrm{Zr}(o$-tolyl $)\left(\mathrm{ClC}_{6} \mathrm{H}_{5}\right)^{+}$, endo isomer $\left(\mathbf{2} \cdot \mathbf{a} \cdot \mathbf{C}_{6} \mathbf{H}_{5} \mathbf{C l}\right.$-endo $)$

$\begin{array}{lrrr}\text { Coordinates } & & & \\ & \mathrm{x} \AA & \mathrm{y} \AA & \mathrm{z} \AA \\ \mathrm{C} & -0.837586000 & -2.372678000 & -1.984119000 \\ \mathrm{C} & -1.730449000 & -1.291293000 & -2.265458000 \\ \mathrm{C} & -2.770722000 & -1.309563000 & -1.289590000 \\ \mathrm{C} & -2.498032000 & -2.380063000 & -0.381429000 \\ \mathrm{C} & -1.301586000 & -3.042164000 & -0.817574000 \\ \mathrm{C} & 0.900977000 & -1.559638000 & 1.809749000 \\ \mathrm{C} & 0.800282000 & -0.136289000 & 1.971668000 \\ \mathrm{C} & -0.528947000 & 0.166665000 & 2.383918000 \\ \mathrm{C} & -1.264950000 & -1.055590000 & 2.429694000 \\ \mathrm{C} & -0.369122000 & -2.124700000 & 2.101678000 \\ \mathrm{C} & 3.167377000 & 0.114264000 & -0.581175000 \\ \mathrm{C} & 3.129254000 & 1.508816000 & -0.491315000 \\ \mathrm{C} & 4.222254000 & 2.152665000 & 0.113040000 \\ \mathrm{C} & 5.309432000 & 1.407832000 & 0.596919000 \\ \mathrm{C} & 5.315685000 & 0.009445000 & 0.479858000 \\ \mathrm{C} & 4.233909000 & -0.660561000 & -0.116470000 \\ \mathrm{Cl} & 1.807832000 & -0.748376000 & -1.395193000 \\ \mathrm{Zr} & -0.660527000 & -0.731154000 & 0.000924000 \\ \mathrm{H} & 0.035947000 & -2.654001000 & -2.575289000 \\ \mathrm{H} & -1.647522000 & -0.590084000 & -3.097093000 \\ \mathrm{H} & -3.625398000 & -0.636139000 & -1.253932000 \\ \mathrm{H} & -3.124849000 & -2.682686000 & 0.458318000 \\ \mathrm{H} & -0.851456000 & -3.926030000 & -0.365380000 \\ \mathrm{H} & 1.798840000 & -2.111234000 & 1.527766000 \\ \mathrm{H} & 1.610784000 & 0.582324000 & 1.840726000 \\ \mathrm{H} & -0.917026000 & 1.159019000 & 2.607214000 \\ \mathrm{H} & -2.310406000 & -1.162642000 & 2.719928000 \\ \mathrm{H} & -0.618168000 & -3.185797000 & 2.090947000 \\ \mathrm{H} & 2.282415000 & 2.081165000 & -0.877703000 \\ \mathrm{H} & 4.219336000 & 3.243640000 & 0.194558000 \\ \mathrm{H} & 6.157011000 & 1.919874000 & 1.061065000 \\ \mathrm{H} & 6.165242000 & -0.572852000 & 0.848450000 \\ \mathrm{H} & 4.234097000 & -1.747877000 & -0.227604000 \\ \mathrm{C} & -1.816502000 & 1.159081000 & -0.045814000 \\ \mathrm{C} & -0.924618000 & 1.763483000 & -0.949266000 \\ \mathrm{C} & -1.024686000 & 3.119982000 & -1.317802000 \\ \mathrm{C} & -2.071387000 & 3.873431000 & -0.773628000 \\ \mathrm{C} & -2.986727000 & 3.275613000 & 0.110066000 \\ \mathrm{C} & -2.879693000 & 1.922303000 & 0.490222000 \\ \mathrm{H} & -0.120255000 & 1.178308000 & -1.450411000 \\ \mathrm{H} & -0.324340000 & 3.561877000 & -2.033471000 \\ \mathrm{H} & -2.189196000 & 4.925724000 & -1.049672000 \\ & & & \\ & & \end{array}$




$\begin{array}{llll}\mathrm{H} & -3.808147000 & 3.877033000 & 0.516210000 \\ \mathrm{C} & -3.880954000 & 1.326396000 & 1.457434000 \\ \mathrm{H} & -4.787862000 & 1.948025000 & 1.532491000 \\ \mathrm{H} & -4.189027000 & 0.311479000 & 1.152332000 \\ \mathrm{H} & -3.461306000 & 1.244799000 & 2.478231000\end{array}$

Vibrational Frequencies $\left(\mathrm{cm}^{-1}\right)$

\begin{tabular}{|c|c|c|c|}
\hline 16.7 & 664.8 & 1060.1 & 2968.9 \\
\hline 23.8 & 667.2 & 1062.0 & 3027.8 \\
\hline 31.3 & 688.2 & 1064.3 & 3066.6 \\
\hline 35.5 & 699.3 & 1067.4 & 3110.0 \\
\hline 47.2 & 727.3 & 1079.0 & 3124.6 \\
\hline 56.0 & 782.3 & 1107.0 & 3132.8 \\
\hline 60.3 & 791.1 & 1121.2 & 3139.5 \\
\hline 78.4 & 797.7 & 1124.2 & 3142.3 \\
\hline 90.8 & 800.2 & 1166.6 & 3149.4 \\
\hline 104.4 & 802.4 & 1167.4 & 3154.3 \\
\hline 118.0 & 806.6 & 1173.9 & 3157.9 \\
\hline 130.9 & 809.0 & 1194.4 & 3172.4 \\
\hline 134.8 & 813.9 & 1251.3 & 3177.2 \\
\hline 157.8 & 821.8 & 1254.8 & 3181.1 \\
\hline 170.1 & 823.3 & 1261.9 & 3183.4 \\
\hline 209.0 & 824.4 & 1300.9 & 3189.6 \\
\hline 216.0 & 826.1 & 1337.1 & 3190.6 \\
\hline 236.0 & 828.3 & 1352.7 & 3197.4 \\
\hline 247.3 & 876.8 & 1369.4 & 3201.1 \\
\hline 249.9 & 879.9 & 1372.3 & 3213.3 \\
\hline 273.2 & 885.1 & 1376.5 & 3214.9 \\
\hline 285.7 & 889.2 & 1382.4 & \\
\hline 300.7 & 893.2 & 1395.5 & \\
\hline 322.7 & 897.6 & 1422.3 & \\
\hline 344.2 & 935.6 & 1433.7 & \\
\hline 386.3 & 951.8 & 1437.1 & \\
\hline 391.6 & 969.5 & 1442.9 & \\
\hline 400.1 & 973.0 & 1445.6 & \\
\hline 419.8 & 974.6 & 1449.4 & \\
\hline 458.8 & 995.1 & 1459.2 & \\
\hline 482.1 & 1007.7 & 1468.4 & \\
\hline 522.4 & 1010.1 & 1469.0 & \\
\hline 564.1 & 1015.2 & 1482.2 & \\
\hline 570.9 & 1018.0 & 1562.0 & \\
\hline 577.0 & 1020.0 & 1575.6 & \\
\hline 580.0 & 1033.8 & 1580.9 & \\
\hline 598.7 & 1051.1 & 1601.6 & \\
\hline 628.9 & 1058.3 & 2912.4 & \\
\hline
\end{tabular}


